\title{
Channel Estimation for Large Intelligent Surface-Based Transceiver Using a Parametric Channel Model
}

This paper was downloaded from TechRxiv (https://www.techrxiv.org).

\section{LICENSE}

CC BY 4.0

SUBMISSION DATE / POSTED DATE

$05-12-2021 / 10-12-2021$

\section{CITATION}

ghermezcheshmeh, mojtaba; Jamali, Vahid; Gacanin,, Haris; zlatanov, Nikola (2021): Channel Estimation for Large Intelligent Surface-Based Transceiver Using a Parametric Channel Model. TechRxiv. Preprint. https://doi.org/10.36227/techrxiv.17126735.v1

$\mathrm{DOI}$

10.36227/techrxiv.17126735.v1 


\title{
Channel Estimation for Large Intelligent
}

\section{Surface-Based Transceiver Using a Parametric Channel Model}

\author{
Mojtaba Ghermezcheshmeh, Vahid Jamali, Member, IEEE, Haris Gacanin, \\ Fellow, IEEE, and Nikola Zlatanov, Member, IEEE
}

\begin{abstract}
Large intelligent surface-based transceivers (LISBTs), in which a spatially continuous surface is being used for signal transmission and reception, have emerged as a promising solution for improving the coverage and data rate of wireless communication systems. To realize these objectives, the acquisition of accurate channel state information (CSI) in LISBT-assisted wireless communication systems is crucial. In this paper, we propose a channel estimation scheme based on a parametric physical channel model for line-of-sight dominated communication in millimeter and terahertz wave bands. The proposed estimation scheme requires only five pilot signals to perfectly estimate the channel parameters assuming there is no noise at the receiver. In the presence of noise, we propose an iterative estimation algorithm that decreases the channel estimation error due to noise. The training overhead and computational cost of the proposed scheme do not scale with the number of antennas. The simulation results demonstrate that the proposed estimation scheme significantly outperforms other benchmark schemes.
\end{abstract}

\section{Index Terms}

Channel estimation, millimeter-wave communication, large intelligent surface-based transceiver, beyond massive MIMO, parametric channel model.

M. Ghermezcheshmeh, and N. Zlatanov are with the Department of Electrical and Computer Systems Engineering, Monash University, Melbourne, VIC 3800, Australia (Emails: mojtaba.ghermezcheshmeh@monash.edu; nikola.zlatanov@monash.edu).

V. Jamali is with the Department of Electrical and Computer Engineering, Princeton University, Princeton, NJ 08544 USA (Email: jamali@princeton.edu).

H. Gacanin is with the Faculty of Electrical Engineering and Information Technology, RWTH Aachen University, Aachen, Germany (Email: harisg@ice.rwth-aachen.de). 


\section{INTRODUCTION}

The millimeter-wave (mmWave) and terahertz (THz) frequency bands will play a pivotal role in next-generation (e.g., beyond 5G) wireless networks since they would be able to provide abundant spectrum resources, higher data rates, and lower latency [1], [2]. However, sever path-loss, atmospheric absorption, human blockage, and other environmental obstruction are key challenges for enabling wireless communication in the mmWave and $\mathrm{THz}$ bands [3], [4]. Over the past few years, massive multiple-input multiple-output (MIMO) communication systems, in which base stations (BSs) are equipped with a large antenna array, have been introduced to provide beamforming gains that can overcome the mentioned limitations [5], [6]. In order to realize massive MIMO BSs with a large antenna array, different architectures such as fully-digital, hybrid analog/digital, and lens array antennas architectures have been proposed [4]. Since the beamforming gain of massive MIMO increases with the number of antennas, it would be highly desirable to have infinitely many antennas ${ }^{1}$, which is not feasible with large antenna arrays [7], [8]. In this direction, large intelligent surfaces (LISs) [9], also known as holographic massive MIMO [8], holographic MIMO surfaces [10], and dynamic metasurface antennas [11], were introduced to go beyond massive MIMO systems [8]-[16].

The LISs have emerged as a promising technology for packing infinitely many antennas into a very small area utilizing sub-wavelength phase-shifting elements [8], [9]. The LISs that comprise a massive number of phase-shifting elements with steerable beamforming weight are used for generating narrow beams with high beamforming gains [11]. The LIS can be used as an intelligent reflecting surface (IRS) or an LIS-based transceiver (LISBT) [8]-[10]. An IRS, which is placed between the BS and the user, manipulates the incident electromagnetic (EM) wave impinging on it and reflects it towards the user [12], [13]. When the direct link between the BS and the user is blocked by an obstacle, deploying an IRS that has a line-of-sight (LoS) link with the BS and the user can bypass the obstacle via intelligent reflection [14]. In contrast to the IRS that has attracted considerable research attention, the application of LIS as a LISBT has lacked behind in research outputs. Thereby, LISBTs are the focus of this paper.

The LISBTs can control the analog beamforming for transmission and reception [9], [15], see Fig. 1. The LISBTs can be seen as an extension of traditional massive MIMO from a discrete

\footnotetext{
${ }^{1}$ The infinite number of antennas is just a theoretical term. We refer here to extremely large number of sub-wavelength phase-shifting elements.
} 


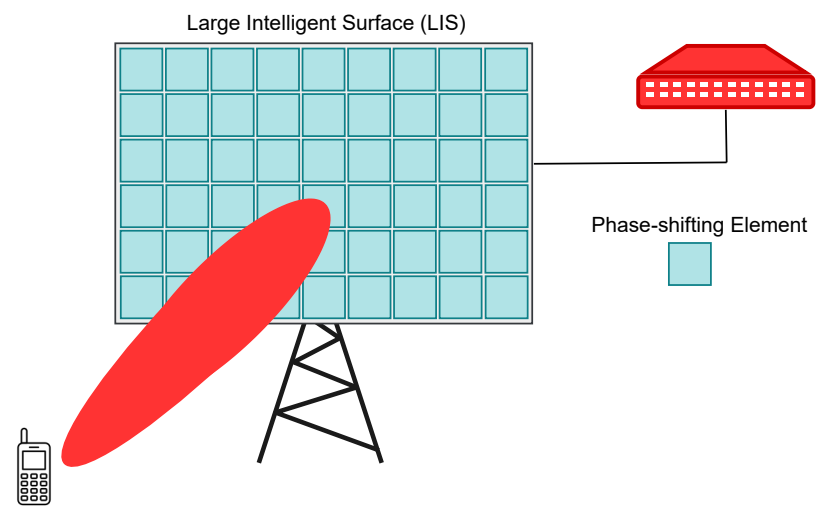

Figure 1. The LISBT, in which the LIS consists of a massive number of phase-shifting elements, transmits/receives the signal with high beamforming gain towards/from the user.

array to a continuous surface [16]. In the LISBT, multiple radio frequency (RF) chains can be connected to one LIS to serve multiple users using spatial multiplexing [8], [11]. The existing research works have verified the effect of LISBTs on enhancing the communication performance in various scenarios under the assumption of the availability of perfect channel state information (CSI) [15]-[18]. To achieve the full potential of the LISBTs, the acquisition of accurate CSI is a fundamental and challenging task in practice. Since the LISBTs consists of a massive number of phase-shifting elements, estimating all the entries of the channel matrix is not feasible in practice since it results in substantial training overhead and computational cost. Instead, thanks to the sparse nature of the channels in mmWave and $\mathrm{THz}$ frequency bands, the parameters of the dominant paths, e.g., angle of arrival (AoA), angle of departure (AoD), and path gain, can be estimated to reduce the training overhead [19]. Motivated by this, in this paper, we propose a channel estimation scheme for LISBT-assisted wireless communication system based on the path parameters that results in reduced training overhead.

\section{A. Related Work}

The channel estimation schemes in the massive MIMO literature can be applied to the LISBTassisted communication systems. Up to now, a variety of channel estimation schemes have been proposed for the massive MIMO communication systems, including exhaustive search [20], hierarchical search [19], [21]-[23], and compressed sensing (CS) [24]-[31]. The authors in [20] propose the exhaustive search algorithm as a straightforward approach where the transmitter and receiver scan all possible angular directions to find the best pair of AoA and AoD. However, 
the training overhead of the exhaustive search approach is prohibitively high, especially when a large number of antennas generate narrow beams in the mmWave and $\mathrm{THz}$ frequency bands. To improve the efficiency of the exhaustive search, the hierarchical search based on a predefined codebook was proposed in [19], [21]-[23]. In the first stage of the hierarchical search, the codewords with larger beam widths are used to scan the entire angular domain. Then, in the second stage, codewords with narrower beam widths are used to scan only a specific range obtained at the first stage. In [19], a hierarchical codebook is designed, where sub-array and deactivation antenna processing techniques were exploited to generate the codebook via closedform expressions. Turning off some antennas is not a good approach since the reduced array gain has an undesirable effect on the performance [21]. The authors in [22] design multi-resolution beamforming sequences to quickly search out the dominant channel direction. Reference [23] proposes a beam training method based on dynamic hierarchical codebook to estimate the mmWave massive MIMO channel with multi-path components. However, the accuracy of the hierarchical search is limited by the codebook size. Moreover, all these hierarchical schemes may incur high training overhead and system latency because they require non-trivial coordination among the transmitter and the receiver.

It is shown in [24]-[31] that CS is also an attractive approach to formulate the channel estimation problem as a sparse signal recovery problem. In the CS-based channel estimation, first a set of random training sequences is used to measure the channel, and then a sparse recovery algorithm is employed to obtain the path parameters. The CS-based channel estimation schemes can be classified into two main categories: on-grid and gridless estimation. The ongrid schemes assume that the AoAs and AoDs are discrete with finite resolutions although the actual angles are continuous in practice [24]-[28]. The accuracy of these schemes depends on the quantization resolution as well as the number of measurements. As a result, the training overhead can be significantly high in order to achieve satisfactory accuracy. On the other hand, the gridless schemes deal directly with the continuous domain without imposing a discrete dictionary [29]-[31]. The gridless schemes increase the accuracy of estimation at the expense of increased computational complexity. None of these channel estimation schemes have been explicitly designed for LISBT-assisted communication systems.

In the user localization problem, the parametric channel model is used to estimate the distance of the user from the BS and the elevation and azimuth AoD [32], [33]. Thereby, the channel estimation problem and user localization are highly correlated [34], [35]. Different from the 
studies on localization problems [36]-[41], in this work, we circumvent the estimation of distance in order to obtain a closed-form solution for the channel estimation problem.

\section{B. Main Contributions}

Different from the aforementioned works, we obtain a closed-form solution for the channel estimation problem based on a parametric channel model. To be more specific, we model the channel based on the path parameters of the system and then estimate those parameters with the lowest possible number of pilots. The training overhead and the computational cost of the proposed scheme do not scale with the the number of antennas. Specifically, our main contributions are as follows.

- Based on the parametric channel model in the far-field, we show that only three parameters are required to be estimated in order to obtain the optimal phase shifts for all phase-shifting elements. These three parameters include the distance from the LISBT to the user and the elevation and azimuth AoD. The distance from the LISBT has to be estimated very precisely, which is not possible in practice. Therefore, we simplify the LISBT-user channel for the far-field region of the LISBT to circumvent the estimation of that parameter. We show that if we use the simplified model, the number of parameters needed to be estimated decreases from three to two.

- For the near-field of the LISBT, we partition the LIS into tiles such that the far-field condition holds for each tile [42]. Then, the channel in the near-field is modeled as the superposition of the channels through the individual tiles. We show that the structure of the channel model in both the far-field and near-field regions is the same. Therefore, we focus on maximizing the received power at the user located in the far-field.

- Finally, we present an efficient scheme to obtain the unknown parameters based on the far-field channel model. In the case without receiver noise, the proposed scheme requires only five pilots in order to perfectly estimate the unknown channel parameters. In the case with receiver noise, we present an iterative algorithm based on the proposed scheme to estimate the unknown channel parameters. Since the performance of the proposed iterative algorithm depends on the initial values of the algorithm, we propose a simple method to provide initial values using three pilot signals.

The remaining part of this paper is organized as follows. In Sec. II, the system model is described. We obtain the optimal phase shifts at the LISBT in Sec. III. In Sec. IV, we provide 


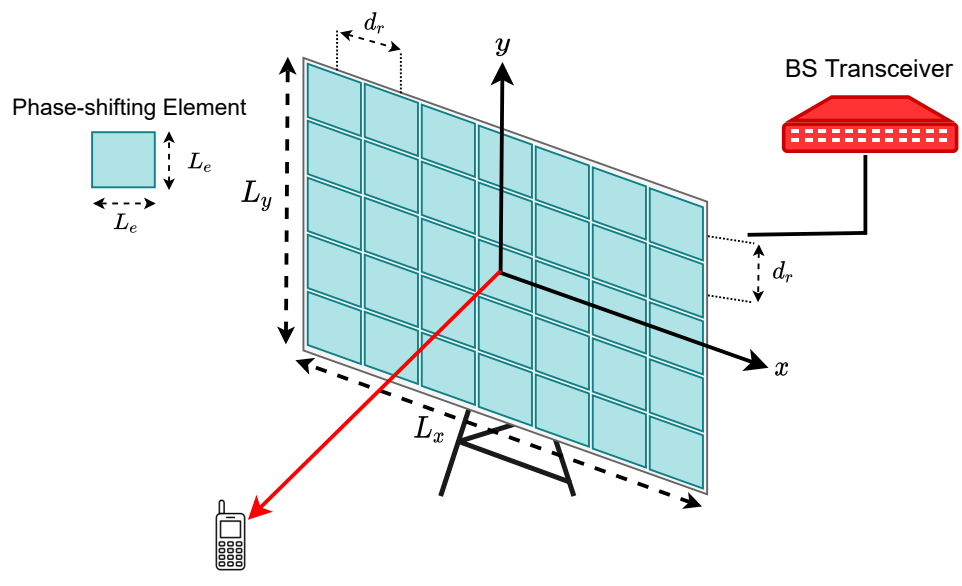

Figure 2. Schematic illustration of the LISBT-assisted wireless communication system. Each phase-shifting element of the LIS changes the phase of the RF signal, and hence the LISBT is able to send a beamformed signal towards the user.

channel models that hold in the far-field and near-field, respectively. Then, we propose our estimation scheme in the case with and without receiver noise in Sec. V. The simulation results are provided in Sec. VI. Finally, Sec. VII concludes the paper.

\section{System Model}

In this section, we introduce the system and channel models.

\section{A. System Model}

As shown in Fig. 2, we consider the LIS as a LISBT that communicates with a user. In the LISBT-assisted wireless communication system, the RF signal is generated at the backside of the LIS, and then it passes through the LIS where each phase-shifting element changes the phase of the passing RF signal. Thereby, the LISBT is able to send a beamformed signal towards the user, see Fig. 2. We focus on the LISBT with only one RF signal generator since the channel estimation problem in a LISBT with one RF signal generator is more challenging than a LISBT with two or more RF signal generators ${ }^{2}$.

We consider the LIS as a rectangular surface of size $L_{x} \times L_{y}$, where $L_{x}$ and $L_{y}$ are the width and the length of the surface, respectively. The LISBT is comprised of a large number of subwavelength phase-shifting elements of size $L_{e} \times L_{e}$ that can change the phase and amplitude of

\footnotetext{
${ }^{2}$ Note that the degree of freedom of the received signals at baseband is limited by the number of RF signal generators [43].
} 
the signal. We assume that the LIS lies in the $x-y$ plane of a Cartesian coordinate system, and the center of the surface is placed at the origin of the coordinate system, see Fig. 2. Let $d_{r}$ be the distance between neighboring phase-shifting elements. The total number of phase-shifting elements of the LISBT is given by $M=M_{x} \times M_{y}$, where $M_{x}=L_{x} / d_{r}$ and $M_{y}=L_{y} / d_{r}$. Assuming $M_{x}$ and $M_{y}$ are odd numbers, the position of the $\left(m_{x}, m_{y}\right)$-th phase-shifting element is given by $(x, y)=\left(m_{x} d_{r}, m_{y} d_{r}\right)$ for $m_{x}=-\frac{M_{x}-1}{2}, \cdots, \frac{M_{x}-1}{2}$ and $m_{y}=-\frac{M_{y}-1}{2}, \cdots, \frac{M_{y}-1}{2}$.

\section{B. Channel Model}

Generally, the channel between two transceivers may consist of one LoS and some non-LoS (NLoS) components. Due to severe path-loss at the mmWave and $\mathrm{THz}$ frequency bands, the power of the LoS component is much higher than the power of the NLoS component [44], [45]. Therefore, we only consider the LoS component to model the channel between the LISBT and the user $^{3}$. In the following, we model the channel between the LISBT and the user based on the path parameters of the system.

For the LISBT-user channel, we start with the channel between the $\left(m_{x}, m_{y}\right)$-th phase-shifting element at the LISBT and the user. Let $t_{m_{x} m_{y}}$ denote the channel coefficient between the $\left(m_{x}, m_{y}\right)$-th phase-shifting element at the LISBT and the user. Due to the LoS assumption, $t_{m_{x} m_{y}}$ is given by [46]

$$
t_{m_{x} m_{y}}=\frac{\lambda \sqrt{F_{m_{x} m_{y}}}}{4 \pi d_{m_{x} m_{y}}} e^{-j k_{0} d_{m_{x} m_{y}}},
$$

where $\lambda$ is the wavelength of the carrier frequency, $k_{0}=2 \pi / \lambda$ is the wave number, $d_{m_{x} m_{y}}$ is the distance between the center of the $\left(m_{x}, m_{y}\right)$-th phase-shifting element at the LISBT and the user, and $F_{m_{x} m_{y}}$ accounts for the effect of the size and power radiation pattern of the phase-shifting elements on the channel coefficient.

Each phase-shifting element can be configured to impose different levels of phase shifts on the transmitted and received signal [11]. Let $\Gamma_{m_{x} m_{y}}=e^{j \beta_{m_{x} m_{y}}}$ denote the beamforming weight of the $\left(m_{x}, m_{y}\right)$-th phase-shifting element at the LISBT, where $\beta_{m_{x} m_{y}}$ is the phase shift at the $\left(m_{x}, m_{y}\right)$-th element. For ease of presentation, let us define the phase shift parameters of the

\footnotetext{
${ }^{3}$ The effect of NLoS components on the proposed scheme will be investigated in the Numerical Results section.
} 
LISBT for all elements as $\boldsymbol{\beta}=\left\{\beta_{m_{x} m_{y}} ; \forall m_{x}, m_{y}\right\}$. The LISBT-user channel, denoted by $H(\boldsymbol{\beta})$, can be written as

$$
\begin{aligned}
H(\boldsymbol{\beta}) & =\sum_{m_{x}=-\frac{M_{x}-1}{2}}^{\frac{M_{x}-1}{2}} \sum_{m_{y}=-\frac{M_{y}-1}{2}}^{\frac{M_{y}-1}{2}} \Gamma_{m_{x} m_{y}} t_{m_{x} m_{y}} \\
& =\frac{\lambda}{4 \pi} \sum_{m_{x}=-\frac{M_{x}-1}{2}}^{\frac{M_{x}-1}{2}} \sum_{m_{y}=-\frac{M_{y}-1}{2}}^{\frac{M_{y}-1}{2}} \frac{\sqrt{F_{m_{x} m_{y}}}}{d_{m_{x} m_{y}}} e^{-j\left(k_{0} d_{m_{x} m_{y}}-\beta_{m_{x} m_{y}}\right) .}
\end{aligned}
$$

From (2), it is clear that the LISBT-user channel depends on the imposed phase shift by each element at the LISBT and the distance of each element from the user.

\section{Optimal Phase Shifts at the LiSBT}

In this section, we present the exact expression for the optimal phase shifts at the LISBT that maximizes the received power at the user.

According to the LISBT-user channel in (2), the received power at the user in the LISBT-assisted wireless communication, denoted by $P_{r}(\boldsymbol{\beta})$, is obtained as

$$
\begin{aligned}
P_{r}(\boldsymbol{\beta}) & =P_{t}|H(\boldsymbol{\beta})|^{2} \\
& =P_{t}\left(\frac{\lambda}{4 \pi}\right)^{2}\left|\sum_{m_{x}=-\frac{M_{x}-1}{2}}^{\frac{M_{x}-1}{2}} \sum_{m_{y}=-\frac{M_{y}-1}{2}}^{\frac{M_{y}-1}{2}} \frac{\sqrt{F_{m_{x} m_{y}}}}{d_{m_{x} m_{y}}} e^{-j\left(k_{0} d_{m_{x} m_{y}}-\beta_{m_{x} m_{y}}\right)}\right|^{2},
\end{aligned}
$$

where $P_{t}$ is the transmission power at the LISBT. From (3), it is clear that the received power at the user is maximized by setting $\beta_{m_{x} m_{y}}$ to

$$
\beta_{m_{x} m_{y}}=\bmod \left(k_{0} d_{m_{x} m_{y}}, 2 \pi\right), \forall m_{x}, m_{y} .
$$

The total number of parameters to be estimated is equal to the total number of phase-shifting elements at the LISBT $M_{x} \times M_{y}$. In the following, we will show that the number of parameters that need to be estimated can be reduced significantly when we obtain a closed-form expression for $d_{m_{x} m_{y}}, \forall m_{x}, m_{y}$.

As shown in Fig. 3, the distance between the center of the LIS to the center of the $\left(m_{x}, m_{y}\right)$-th phase-shifting element is $d_{r} \sqrt{m_{x}^{2}+m_{y}^{2}}$. Let $d_{0}$ denote the distance between the user and the center of the LIS, see Fig. 3. In addition, let $\delta_{m_{x}, m_{y}}$ denote the angle between the vector from the center of the LIS to the user and the vector from the center of the LIS to the center of the 


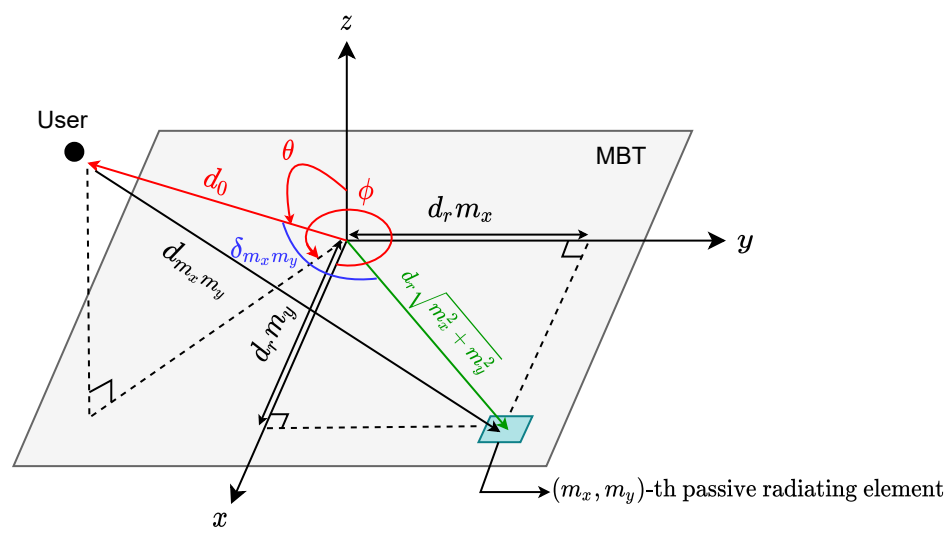

Figure 3. Using the cosin rule to obtain the distance between the $\left(m_{x}, n_{y}\right)$-th phase-shifting element at the LISBT to the user.

$(m, n)$-th phase-shifting element, see Fig. 3. Now, we can use the cosine rule to obtain $d_{m_{x} m_{y}}$ as a function of $d_{r}, m_{x}, m_{y}$, and $\delta_{m_{x} m_{y}}$ as

$$
d_{m_{x} m_{y}}=\left(d_{0}^{2}+d_{r}^{2}\left(m_{x}^{2}+m_{y}^{2}\right)-2 d_{0} d_{r} \sqrt{m_{x}^{2}+m_{y}^{2}} \cos \left(\delta_{m_{x} m_{y}}\right)\right)^{\frac{1}{2}} .
$$

Let $\theta \in[0, \pi / 2]$ and $\phi \in[0,2 \pi]$ denote the elevation and azimuth angles of the impinging wave from the user to the center of the LIS, see Fig. 3. Therefore, the vector from the center of the LIS to the user can be obtain as $\left[d_{0} \sin (\theta) \cos (\phi), d_{0} \sin (\theta) \sin (\phi), d_{0} \cos (\theta)\right]$, and the vector from the center of the LIS to the center of the $\left(m_{x}, m_{y}\right)$-th phase-shifting element is $\left[d_{r} m_{x}, d_{r} m_{y}, 0\right]$. According to the inner product between these two vectors, we can write $\cos \left(\delta_{m_{x}, m_{y}}\right)$ as

$$
\begin{aligned}
\cos \left(\delta_{m_{x} m_{y}}\right) & =\frac{\left[d_{0} \sin (\theta) \cos (\phi), d_{0} \sin (\theta) \sin (\phi), d_{0} \cos (\theta)\right] \cdot\left[d_{r} m_{x}, d_{r} m_{y}, 0\right]}{d_{0} d_{r} \sqrt{m_{x}^{2}+m_{y}^{2}}} \\
& =\frac{m_{x} \sin (\theta) \cos (\phi)+m_{y} \sin (\theta) \sin (\phi)}{\sqrt{m_{x}^{2}+m_{y}^{2}}} .
\end{aligned}
$$

Substituting (6) into (5), we obtain a much simpler expression for $d_{m_{x} m_{y}}$ as

$$
d_{m_{x} m_{y}}=\left(d_{0}^{2}+d_{r}^{2}\left(m_{x}^{2}+m_{y}^{2}\right)-2 d_{0} d_{r}\left(m_{x} \alpha_{1}+m_{y} \alpha_{2}\right)\right)^{\frac{1}{2}}
$$

where

$$
\begin{aligned}
& \alpha_{1}=\sin (\theta) \cos (\phi), \\
& \alpha_{2}=\sin (\theta) \sin (\phi) .
\end{aligned}
$$


Substituting (7) into (4), we obtain the final expression for the optimal phase shift at the $\left(m_{x}, m_{y}\right)$ th element of the LISBT that maximizes the LISBT-user channel as

$$
\beta_{m_{x} m_{y}}=\bmod \left(k_{0}\left(d_{0}^{2}+d_{r}^{2}\left(m_{x}^{2}+m_{y}^{2}\right)-2 d_{0} d_{r}\left(m_{x} \alpha_{1}+m_{y} \alpha_{2}\right)\right)^{\frac{1}{2}}, 2 \pi\right), \forall m_{x}, m_{y} .
$$

From (10), we conclude that in order to obtain the optimal phase shift at the LISBT, we need to estimate only three parameters, $d_{0}, \alpha_{1}$, and $\alpha_{2}$. Therefore, using (10) reduces the number of parameters from $M_{x} \times M_{y}$ to three. However, $d_{0}$ has to be estimated very precisely in order to set the optimal phase shifts, which is not possible in practice. In the following, we circumvent the estimation of $d_{0}$ such that $\alpha_{1}$ and $\alpha_{2}$ are the only two parameters left to estimate.

\section{FAR-FiEld And NeAR-FiEld Channel Models}

In this section, we provide the LISBT-user channel models for the far-field and the near-field regions. We will use these models in Sec. V to develop our channel estimation scheme.

\section{A. Far-Field Channel Model}

For the sake of completeness, we follow the same approach as [47, Ch. 6] to simplify the LISBT-user channel in (2) such that it holds for the far-field region of the LISBT. In the antenna array terminology, the far-field is referred to as the condition that the maximum phase error of the received signal on the antenna array does not exceed $\frac{\pi}{8}$ [48].

The distance between the user and the $\left(m_{x}, m_{y}\right)$-th phase-shifting element at the LISBT in (7) can be rewritten as

$$
d_{m_{x} m_{y}}=d_{0}\left(1+\left(\frac{d_{r}^{2}\left(m_{x}^{2}+m_{y}^{2}\right)}{d_{0}^{2}}-\frac{2 d_{r}\left(m_{x} \alpha_{1}+m_{y} \alpha_{2}\right)}{d_{0}}\right)\right)^{\frac{1}{2}} .
$$

Now, we can use the first-order Taylor approximation [49], i.e., $\sqrt{1+x} \approx 1+\frac{x}{2}$, to approximate $d_{m_{x} m_{y}}$ in (11) as

$$
d_{m_{x} m_{y}} \approx d_{0}-d_{r}\left(m_{x} \alpha_{1}+m_{y} \alpha_{2}\right)+\frac{d_{r}^{2}\left(m_{x}^{2}+m_{y}^{2}\right)}{2 d_{0}} .
$$

Substituting (12) into (2), the LISBT-user channel in the far-field region of the LISBT, denoted by $H^{(f f)}(\boldsymbol{\beta})$, is then approximated as

$$
\begin{array}{r}
H^{(f f)}(\boldsymbol{\beta}) \approx \frac{\lambda}{4 \pi} \sum_{m_{x}=-\frac{M_{x}-1}{2} m_{m_{y}=-\frac{M_{y}-1}{2}}}^{\frac{M_{x}-1}{2}} \frac{\sqrt{F_{m_{x} m_{y}}}}{\left(d_{0}-d_{r}\left(m_{x} \alpha_{1}+m_{y} \alpha_{2}\right)+\frac{d_{r}^{2}\left(m_{x}^{2}+m_{y}^{2}\right)}{2 d_{0}}\right)} \\
\times e^{-j\left(k_{0}\left(d_{0}-d_{r}\left(m_{x} \alpha_{1}+m_{y} \alpha_{2}\right)+\frac{d_{r}^{2}\left(m_{x}^{2}+m_{y}^{2}\right)}{2 d_{0}}\right)-\beta_{m_{x} m_{y}}\right) .} .
\end{array}
$$


When $\frac{d_{r}^{2}\left(m_{x}^{2}+m_{y}^{2}\right)}{2 d_{0}}$ in the argument of the exponential term in (13) is much smaller than $2 \pi$, we can neglect its impact. Neglecting this term in (13) leads to the following maximum phase error

$$
\underset{m_{x}, m_{y}}{\operatorname{Maximize}} k_{0}\left(\frac{d_{r}^{2}\left(m_{x}^{2}+m_{y}^{2}\right)}{2 d_{0}}\right)=\frac{k_{0}\left(\frac{L_{x}^{2}}{4}+\frac{L_{y}^{2}}{4}\right)}{2 d_{0}}=\frac{k_{0} D_{\mathrm{ITS}}^{2}}{8 d_{0}},
$$

where $D_{\text {ITS }}$ is the diagonal of the LIS. Assuming a maximum phase error of $\frac{\pi}{8}$, the far-field region of the LISBT is obtained as

$$
\frac{2 \pi}{\lambda} \times \frac{D_{\mathrm{ITS}}^{2}}{8 d_{0}} \leq \frac{\pi}{8}
$$

which leads to

$$
d_{0} \geq \frac{2 D_{\mathrm{ITS}}^{2}}{\lambda}
$$

Neglecting $\frac{d_{r}^{2}\left(m_{x}^{2}+m_{y}^{2}\right)}{2 d_{0}}$ in the argument of the exponential term in (13), we have

$$
H^{(f f)}(\boldsymbol{\beta}) \approx \frac{\lambda e^{-j k_{0} d_{0}}}{4 \pi} \sum_{m_{x}=-\frac{M_{x}-1}{2}}^{\frac{M_{x}-1}{2}} \sum_{m_{y}=-\frac{M_{y}-1}{2}}^{\frac{M_{y}-1}{2}} \frac{\sqrt{F_{m_{x} m_{y}}} e^{j\left(k_{0} d_{r}\left(m_{x} \alpha_{1}+m_{y} \alpha_{2}\right)+\beta_{m_{x} m_{y}}\right)}}{\left(d_{0}-d_{r}\left(m_{x} \alpha_{1}+m_{y} \alpha_{2}\right)\right)} .
$$

According to (16), since the size of the LIS is much smaller than $d_{0}$, we can approximate $\frac{1}{\left(d_{0}-d_{r}\left(m_{x} \alpha_{1}+m_{y} \alpha_{2}\right)\right)}$ with $\frac{1}{d_{0}}$. In addition, since the radiation power pattern of all elements in the far-field region of the LISBT is the same, we can write $F_{m_{x} m_{y}}=F$. Therefore, the LISBT-user channel in the far-field of the LISBT can be written as

$$
H^{(f f)}(\boldsymbol{\beta}) \approx \frac{\sqrt{F} \lambda e^{-j k_{0} d_{0}}}{4 \pi d_{0}} \sum_{m_{x}=-\frac{M_{x}-1}{2}}^{\frac{M_{x}-1}{2}} \sum_{m_{y}=-\frac{M_{y}-1}{2}}^{\frac{M_{y}-1}{2}} e^{j\left(k_{0} d_{r}\left(m_{x} \alpha_{1}+m_{y} \alpha_{2}\right)+\beta_{m_{x} m_{y}}\right)} .
$$

It can be observed from (18) that $H^{(f f)}(\boldsymbol{\beta})$ attains its maximum value when we set

$$
\beta_{m_{x} m_{y}}=-\bmod \left(k_{0} d_{r}\left(m_{x} \alpha_{1}+m_{y} \alpha_{2}\right), 2 \pi\right), \forall m_{x}, m_{y}
$$

From (19), we can conclude that in order to obtain the optimal phase shift for maximizing the LISBT-user channel in the far-field region of the LISBT, we need to estimate only two parameters, $\alpha_{1}$ and $\alpha_{2}$.

Since we do not have the values of $\alpha_{1}$ and $\alpha_{2}$, we consider a general case to see what would be the LISBT-user channel in the far-field of the LISBT if we replace $\alpha_{1}$ and $\alpha_{2}$ with any other values. In the following lemma, we apply a linear phase shift to each phase-shifting element to obtain a closed-form expression for the LISBT-user channel in the far-field of the LISBT. 
Lemma 1. If we apply the following linear phase shift across the LISBT elements, i.e., to the $\left(m_{x}, m_{y}\right)$-th element

$$
\beta_{m_{x} m_{y}}=-\bmod \left(k_{0} d_{r}\left(m_{x} \beta_{1}+m_{y} \beta_{2}\right), 2 \pi\right), \forall m_{x}, m_{y},
$$

then, the LISBT-user channel in the far-field of the LISBT is a function of $\beta_{0}, \beta_{1}$, and $\beta_{2}$, and can be obtained as

$$
H^{(f f)}\left(\beta_{0}, \beta_{1}, \beta_{2}\right) \approx\left(\frac{\sqrt{F} \lambda e^{-j k_{0} d_{0}}}{4 \pi d_{0}}\right) \frac{\sin \left(\frac{k_{0} L_{x}}{2}\left(\alpha_{1}-\beta_{1}\right)\right)}{\sin \left(\frac{k_{0} d_{r}}{2}\left(\alpha_{1}-\beta_{1}\right)\right)} \times \frac{\sin \left(\frac{k_{0} L_{y}}{2}\left(\alpha_{2}-\beta_{2}\right)\right)}{\sin \left(\frac{k_{0} d_{r}}{2}\left(\alpha_{2}-\beta_{2}\right)\right)} .
$$

Proof. The proof follows similar steps as [42, and 47, Ch. 6] and is provided in Appendix A for completeness.

For the extremely sub-wavelength elements $\left(d_{r} \rightarrow 0\right)$, the LIS acts as a continuous surface [10]. Assuming $d_{r} \rightarrow 0$, we can approximate $\sin \left(\frac{k_{0} d_{r}}{2}\left(\alpha_{1}-\beta_{1}\right)\right)$ and $\sin \left(\frac{k_{0} d_{r}}{2}\left(\alpha_{2}-\beta_{2}\right)\right)$ in the denominator of (21) with $\frac{k_{0} d_{r}}{2}\left(\alpha_{1}-\beta_{1}\right)$ and $\frac{k_{0} d_{r}}{2}\left(\alpha_{2}-\beta_{2}\right)$, respectively. Then, the LISBT-user channel in the far-field of the LISBT in (21) can be written as

$$
H^{(f f)}\left(\beta_{0}, \beta_{1}, \beta_{2}\right) \approx\left(\frac{\sqrt{F} \lambda e^{-j k_{0} d_{0}}}{4 \pi d_{0}}\right) M_{x} M_{y} \operatorname{sinc}\left(\frac{k_{0} L_{x}}{2}\left(\alpha_{1}-\beta_{1}\right)\right) \operatorname{sinc}\left(\frac{k_{0} L_{y}}{2}\left(\alpha_{2}-\beta_{2}\right)\right)
$$

where $\operatorname{sinc}(x)=\frac{\sin (x)}{x}$. According to (22), the absolute value of the LISBT-user channel would be maximized when the sinc functions attain their maximum value, which occurs for the first and second sinc functions when $\beta_{1}$ and $\beta_{2}$ are set as $\beta_{1}=\alpha_{1}$ and $\beta_{2}=\alpha_{2}$, respectively. Therefore, when we use the far-field channel model, we only need to estimate two parameters, $\alpha_{1}$ and $\alpha_{2}$. Compared with the optimal phase shift in (10), we observe that the number of parameters needed to be estimated decreases from three to two. Most importantly, we have avoided the estimation of $d_{0}$, which was the problematic parameter for estimation.

\section{B. Near-Field Channel Model}

In this subsection, we will present a model for the LISBT-user channel in (2) that holds in the near-field region. According to (16), the near-field region of the LISBT is given by

$$
d_{0} \leq \frac{2 D_{\mathrm{ITS}}^{2}}{\lambda}
$$

Similar to [42], we partition the LIS into tiles which are small enough to satisfy the far-field condition in (16). Different tiles can jointly configure their phase-shifting elements to maximize 


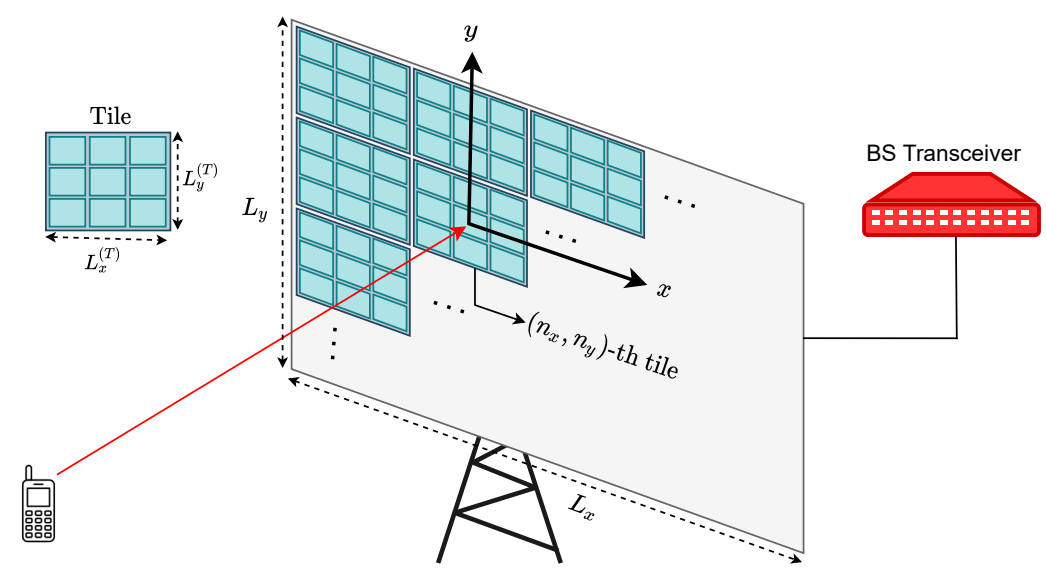

Figure 4. The LIS is partitioned into tiles such that the far-field condition holds for each tile.

the power at the intended receiver. As shown in Fig. 4, we assume that the LISBT is partitioned into $N_{x} \times N_{y}$ tiles of size $L_{x}^{(\mathrm{T})} \times L_{y}^{(\mathrm{T})}$, where $L_{x}^{(\mathrm{T})}=L_{x} / N_{x}$ and $L_{y}^{(\mathrm{T})}=L_{y} / N_{y}$ are the width and the length of the tile, respectively. The total number of phase-shifting elements of each tile is given by $M^{(\mathrm{T})}=M_{x}^{(\mathrm{T})} \times M_{y}^{(\mathrm{T})}$, where $M_{x}^{(\mathrm{T})}=L_{x}^{(\mathrm{T})} / d_{r}$ and $M_{y}^{(\mathrm{T})}=L_{y}^{(\mathrm{T})} / d_{r}$. Assuming $N_{x}$ and $N_{y}$ are odd numbers, the position of the center of the $\left(n_{x}, n_{y}\right)$-th tile is given by $(x, y)=\left(n_{x} L_{x}^{(T)}, n_{y} L_{y}^{(T)}\right)$ for $n_{x}=-\frac{N_{x}-1}{2}, \cdots, \frac{N_{x}-1}{2}$ and $n_{y}=-\frac{N_{y}-1}{2}, \cdots, \frac{N_{y}-1}{2}$.

According to the far-field condition in (16), we can use the far-field channel model between a given tile of the LISBT and the user if the following condition holds

$$
d_{0} \geq \frac{2 D_{\mathrm{T}}^{2}}{\lambda}
$$

where $D_{\mathrm{T}}$ is the diagonal of the tile. Substituting $D_{\mathrm{T}}=\sqrt{\left(\frac{L_{x}}{N_{x}}\right)^{2}+\left(\frac{L_{y}}{N_{y}}\right)^{2}}$ into (24), we can obtain $N_{x}$ and $N_{y}$ from the following inequality

$$
d_{0} \geq \frac{2\left(\left(\frac{L_{x}}{N_{x}}\right)^{2}+\left(\frac{L_{y}}{N_{y}}\right)^{2}\right)}{\lambda} .
$$

We first focus on the channel between the $\left(n_{x}, n_{y}\right)$-th tile of the LISBT and the user. We assume that the center of the $\left(n_{x}, n_{y}\right)$-th tile is placed at the origin of the coordinate system. Let $\theta_{n_{x} n_{y}}$ and $\phi_{n_{x} n_{y}}$ denote the elevation and azimuth angles of the impinging wave from the user to the center of the $\left(n_{x}, n_{y}\right)$-th tile of the LISBT. Similar to (8) and (9), let us define $\alpha_{1_{n_{x} n_{y}}}=\sin \left(\theta_{n_{x} n_{y}}\right) \cos \left(\phi_{n_{x} n_{y}}\right), \alpha_{2_{n_{x} n_{y}}}=\sin \left(\theta_{n_{x} n_{y}}\right) \sin \left(\phi_{n_{x} n_{y}}\right)$, for the $\left(n_{x}, n_{y}\right)$-th tile at the LISBT. Moreover, let $d_{n_{x} n_{y}}^{(\mathrm{T})}$ denote the distance between the center of the $\left(n_{x}, n_{y}\right)$-th tile of the 
LISBT and the user, see Fig. 4. Assuming $M_{x}^{(\mathrm{T})}$ and $M_{y}^{(\mathrm{T})}$ are odd numbers, the position of the $\left(m_{x}, m_{y}\right)$-th phase-shifting element of the $\left(n_{x}, n_{y}\right)$-th tile is given by $(x, y)=\left(m_{x} d_{r}, m_{y} d_{r}\right)$ for $m_{x}=-\frac{M_{x}^{(\mathrm{T})}-1}{2}, \cdots, \frac{M_{x}^{(\mathrm{T})}-1}{2}$ and $m_{y}=-\frac{M_{y}^{(\mathrm{T})}-1}{2}, \cdots, \frac{M_{y}^{(\mathrm{T})}-1}{2}$. Similar to Lemma 1, if we apply the following linear phase shift to the $\left(m_{x}, m_{y}\right)$-th phase-shifting element of the $\left(n_{x}, n_{y}\right)$-th tile of the LISBT

$$
\beta_{m_{x} m_{y}}^{n_{x} n_{y}}=-\bmod \left(k_{0}\left(m_{x} \beta_{1_{n_{x} n_{y}}}+m_{y} \beta_{2_{n_{x} n_{y}}}\right)+\beta_{0_{n_{x} n_{y}}}, 2 \pi\right) \forall m_{x}, m_{y}
$$

then, the channel between the $\left(n_{x}, n_{y}\right)$-th tile of the LISBT and the user, denoted by $H_{n_{x} n_{y}}\left(\beta_{0_{n_{x} n_{y}}}\right.$, $\left.\beta_{1_{n_{x} n_{y}}}, \beta_{2_{n_{x} n_{y}}}\right)$, can be obtained as

$$
\begin{aligned}
H_{n_{x} n_{y}}\left(\beta_{0_{n_{x} n_{y}}}, \beta_{1_{n_{x} n_{y}}}, \beta_{2_{n_{x} n_{y}}}\right) \approx\left(\frac{\left.\sqrt{F_{n_{x} n_{y}}} \lambda e^{-j k_{0} d_{n_{x} n_{y}}^{(\mathrm{T})}}\right) M_{x}^{(\mathrm{T})} M_{y}^{(\mathrm{T})} \times}{4 \pi d_{n_{x} n_{y}}^{(\mathrm{T})}}\right. \\
\operatorname{sinc}\left(\frac{k_{0} L_{x}^{(\mathrm{T})}}{2}\left(\alpha_{1_{n_{x} n_{y}}}-\beta_{1_{n_{x} n_{y}}}\right)\right) \operatorname{sinc}\left(\frac{k_{0} L_{y}^{(\mathrm{T})}}{2}\left(\alpha_{2_{n_{x} n_{y}}}-\beta_{2_{n_{x} n_{y}}}\right)\right) e^{-j \beta_{0_{n_{x} n_{y}}}},
\end{aligned}
$$

where $\beta_{0_{n_{x} n_{y}}}$ determines the phase of the channel [42] and $F_{n_{x} n_{y}}$ is the radiation power pattern of the phase-shifting elements of $\left(n_{x}, n_{y}\right)$-th tile.

For ease of presentation, let us define the phase shift parameters of the LISBT for all tiles as $\left(\boldsymbol{\beta}_{\mathbf{0}}, \boldsymbol{\beta}_{\mathbf{1}}, \boldsymbol{\beta}_{\mathbf{2}}\right)=\left\{\left(\beta_{0_{n_{x} n_{y}}}, \beta_{1_{n_{x} n_{y}}}, \beta_{2_{n_{x} n_{y}}}\right) ; \forall n_{x}, n_{y}\right\}$. The LISBT-user channel in the near-field region of the LISBT, denoted by $H^{(n f)}\left(\boldsymbol{\beta}_{\mathbf{0}}, \boldsymbol{\beta}_{\mathbf{1}}, \boldsymbol{\beta}_{\mathbf{2}}\right)$, is the superposition of the channels through individual tiles and is obtained as

$$
\begin{aligned}
& H^{(n f)}\left(\boldsymbol{\beta}_{\mathbf{0}}, \boldsymbol{\beta}_{\mathbf{1}}, \boldsymbol{\beta}_{\mathbf{2}}\right) \approx \sum_{n_{x}=-\frac{N_{x}-1}{2}}^{\frac{N_{x}-1}{2}} \sum_{n_{y}=-\frac{N_{y}-1}{2}}^{\frac{N_{y}-1}{2}} H_{n_{x} n_{y}}\left(\beta_{0_{n_{x} n_{y}}}, \beta_{1_{n_{x} n_{y}}}, \beta_{2_{n_{x} n_{y}}}\right) \\
& \approx \sum_{n_{x}=-\frac{N_{x}-1}{2}}^{\frac{N_{x}-1}{2}} \sum_{n_{y}=-\frac{N_{y}-1}{2}}^{\frac{N_{y}-1}{2}}\left(\frac{\sqrt{F} \lambda e^{-j k_{0} d_{n_{x}}^{(\mathrm{T})} n_{y}}}{4 \pi d_{n_{x} n_{y}}^{(\mathrm{T})}}\right) M_{x}^{(\mathrm{T})} M_{y}^{(\mathrm{T})} \\
& \times \operatorname{sinc}\left(\frac{k_{0} L_{x}^{(\mathrm{T})}}{2}\left(\alpha_{1_{n_{x} n_{y}}}-\beta_{1_{n_{x} n_{y}}}\right)\right) \operatorname{sinc}\left(\frac{k_{0} L_{y}^{(\mathrm{T})}}{2}\left(\alpha_{2_{n_{x} n_{y}}}-\beta_{2_{n_{x} n_{y}}}\right)\right) e^{-j \beta_{0_{n_{x} n_{y}}}} .
\end{aligned}
$$

According to (28), in order to maximize the LISBT-user channel in the near-field region, first we need to maximize the channel between the $\left(n_{x}, n_{y}\right)$-th tile of the LISBT and the user in (27), i.e., $H_{n_{x} n_{y}}\left(\beta_{0_{n_{x} n_{y}}}, \beta_{1_{n_{x} n_{y}}}, \beta_{2_{n_{x} n_{y}}}\right), \forall n_{x}, n_{y}$. In order to maximize $H_{n_{x} n_{y}}\left(\beta_{0_{n_{x} n_{y}}}, \beta_{1_{n_{x} n_{y}}}, \beta_{2_{n_{x} n_{y}}}\right)$ for the $\left(n_{x}, n_{y}\right)$-th tile, we need to estimate two parameters, $\alpha_{1_{n_{x} n_{y}}}$ and $\alpha_{2_{n_{x} n_{y}}}$. Finally, $\beta_{0_{n_{x} n_{y}}}$ $\forall n_{x}, n_{y}$ in (28) can be obtained such that the channels through the individual tiles have the same phase. Therefore, since the number of tiles is $N_{x} N_{y}$, we need to estimate $2 N_{x} N_{y}$ parameters for the near-filed region of the LISBT and we have avoided the problematic estimation of $d_{0}$. 


\section{Proposed Channel Estimation}

Now that we have identified the parameters that need to be estimated in the far-field and nearfield regions, we move towards proposing our scheme for their estimation. Specifically, to obtain some intuition, we first propose the channel estimation scheme under the assumption that there is no noise in the system. Then, we propose an iterative algorithm for the channel estimation in the presence of the noise. Since we have decomposed the near-field as a superposition of the far-field, we only present the channel estimation scheme for the far-field scenario.

\section{A. Channel Estimation in the Absence of Noise}

In the channel estimation procedure, the user sends a pilot signal $x_{p}=\sqrt{P_{p}}$ to the LISBT, where $P_{p}$ is the pilot transmit power. In the absence of the noise, the received signal at the LISBT is given by

$$
y\left(\beta_{1}, \beta_{2}\right)=\sqrt{P_{p}} \times H^{(f f)}\left(\beta_{1}, \beta_{2}\right) .
$$

Substituting (22) into (29) and assuming $L_{x}=K_{x} \lambda$ and $L_{y}=K_{y} \lambda$, where $K_{x}, K_{y} \in \mathbb{N}$ are integer numbers, the absolute value of the received signal at the LISBT is given by

$$
\left|y\left(\beta_{1}, \beta_{2}\right)\right| \approx \sqrt{P_{p}}\left(\frac{\sqrt{F} \lambda}{4 \pi d_{0}}\right)\left|M_{x} M_{y} \operatorname{sinc}\left(K_{x} \pi\left(\alpha_{1}-\beta_{1}\right)\right) \operatorname{sinc}\left(K_{y} \pi\left(\alpha_{2}-\beta_{2}\right)\right)\right| .
$$

In the following, we show that $\alpha_{1}$ and $\alpha_{2}$ can be estimated using five pilots sent by the user. Before sending each pilot, the LISBT applies a new phase shift to the elements by changing $\beta_{1}$ and $\beta_{2}$ in (20). For the first pilot, the LISBT sets $\beta_{1}=\hat{\beta}_{1}$ and $\beta_{2}=\hat{\beta}_{2}$, where $\hat{\beta}_{1}$ and $\hat{\beta}_{2}$ are two random numbers in the range of -1 to 1 . Note that, according to (8) and (9), $\alpha_{1}$ and $\alpha_{2}$ are in the range of -1 to 1 . Then, the absolute value of the received signal at the LISBT due to the first pilot signal is given by

$$
\left|y\left(\hat{\beta}_{1}, \hat{\beta}_{2}\right)\right| \approx \sqrt{P_{p}}\left(\frac{\sqrt{F} \lambda}{4 \pi d_{0}}\right)\left|M_{x} M_{y} \operatorname{sinc}\left(K_{x} \pi\left(\alpha_{1}-\hat{\beta}_{1}\right)\right) \operatorname{sinc}\left(K_{y} \pi\left(\alpha_{2}-\hat{\beta}_{2}\right)\right)\right| .
$$

For the second pilot, the LISBT sets $\beta_{1}=\hat{\beta}_{1}+v$ and $\beta_{2}=\hat{\beta}_{2}$, where the value of $v$ will be discussed later. Therefore, the absolute value of the received signal at the LISBT is

$$
\left|y\left(\hat{\beta}_{1}+v, \hat{\beta}_{2}\right)\right| \approx \sqrt{P_{p}}\left(\frac{\sqrt{F} \lambda}{4 \pi d_{0}}\right)\left|M_{x} M_{y} \operatorname{sinc}\left(K_{x} \pi\left(\alpha_{1}-\hat{\beta}_{1}-v\right)\right) \operatorname{sinc}\left(K_{y} \pi\left(\alpha_{2}-\hat{\beta}_{2}\right)\right)\right| \text {. }
$$


If the LISBT divides (31) by (32), it will obtain

$$
\frac{\left|y\left(\hat{\beta}_{1}, \hat{\beta}_{2}\right)\right|}{\left|y\left(\hat{\beta}_{1}+v, \hat{\beta}_{2}\right)\right|} \approx \frac{\left|\operatorname{sinc}\left(K_{x} \pi\left(\alpha_{1}-\hat{\beta}_{1}\right)\right)\right|}{\left|\operatorname{sinc}\left(K_{x} \pi\left(\alpha_{1}-\hat{\beta}_{1}-v\right)\right)\right|}=\frac{\left|\frac{\sin \left(K_{x} \pi\left(\alpha_{1}-\hat{\beta}_{1}\right)\right)}{K_{x} \pi\left(\alpha_{1}-\hat{\beta}_{1}\right)}\right|}{\left|\frac{\sin \left(K_{x} \pi\left(\alpha_{1}-\hat{\beta}_{1}-v\right)\right)}{K_{x} \pi\left(\alpha_{1}-\hat{\beta}_{1}-v\right)}\right|} .
$$

If $v$ is selected such that $K_{x} v \in \mathbb{N}$, we have $\sin \left(K_{x} \pi\left(\alpha_{1}-\hat{\beta}_{1}-v\right)\right)=\sin \left(K_{x} \pi\left(\alpha_{1}-\hat{\beta}_{1}\right)\right)$. Then, (33) can be simplified to

$$
\frac{\left|y\left(\hat{\beta}_{1}, \hat{\beta}_{2}\right)\right|}{\left|y\left(\hat{\beta}_{1}+v, \hat{\beta}_{2}\right)\right|} \approx\left|\frac{\alpha_{1}-\hat{\beta}_{1}-v}{\alpha_{1}-\hat{\beta}_{1}}\right| .
$$

From equation (34), two solutions for $\alpha_{1}$, denoted by $\alpha_{1}^{(1)}$ and $\alpha_{1}^{(2)}$, can be obtained as

$$
\alpha_{1}^{(1) /(2)}=\hat{\beta}_{1}+\frac{\left|y\left(\hat{\beta}_{1}+v, \hat{\beta}_{2}\right)\right|}{\left|y\left(\hat{\beta}_{1}+v, \hat{\beta}_{2}\right)\right| \pm\left|y\left(\hat{\beta}_{1}, \hat{\beta}_{2}\right)\right|} v .
$$

In order to identify the correct solution for $\alpha_{1}$, the third pilot signal should be sent from the user. For the third pilot signal, the LISBT sets $\beta_{1}=\hat{\beta}_{1}-v$ and $\beta_{2}=\hat{\beta}_{2}$. Using the received signal of the first and third pilot signals, two other solutions for $\alpha_{1}$, denoted by $\alpha_{1}^{(3)}$ and $\alpha_{1}^{(4)}$, can be similarly obtained as

$$
\alpha_{1}^{(3) /(4)}=\hat{\beta}_{1}+\frac{\left|y\left(\hat{\beta}_{1}-v, \hat{\beta}_{2}\right)\right|}{-\left|y\left(\hat{\beta}_{1}-v, \hat{\beta}_{2}\right)\right| \pm\left|y\left(\hat{\beta}_{1}, \hat{\beta}_{2}\right)\right|} v .
$$

One of the solutions in (35) is approximately the same as one of the solutions in (36). Therefore, using (35) and (36), the correct solution for $\alpha_{1}$ can be obtained as

$$
\alpha_{1} \approx\left\{\frac{\alpha_{1}^{(i)}+\alpha_{1}^{(j)}}{2}\left|\min _{i, j}\right| \alpha_{1}^{(i)}-\alpha_{1}^{(j)} \mid ; i \in\{1,2\}, j \in\{3,4\}\right\} .
$$

In order to obtain $\alpha_{2}$, two more pilot signals are needed to be sent by the user. For these two pilot signals, the LISBT sets two different phase shifts as $\left(\beta_{1}, \beta_{2}\right)=\left\{\left(\hat{\beta}_{1}, \hat{\beta}_{2}+w\right),\left(\hat{\beta}_{1}, \hat{\beta}_{2}-w\right)\right\}$, where $w$ is selected such that $K_{y} w \in \mathbb{N}$. Then, similar to $\alpha_{1}$, we have the following solutions for $\alpha_{2}$

$$
\begin{aligned}
\alpha_{2}^{(1) /(2)} & =\hat{\beta}_{2}+\frac{\left|y\left(\hat{\beta}_{1}, \hat{\beta}_{2}+w\right)\right|}{\left|y\left(\hat{\beta}_{1}, \hat{\beta}_{2}+w\right)\right| \pm\left|y\left(\hat{\beta}_{1}, \hat{\beta}_{2}\right)\right|} w, \\
\alpha_{2}^{(3) /(4)} & =\hat{\beta}_{2}+\frac{\left|y\left(\hat{\beta}_{1}, \hat{\beta}_{2}-w\right)\right|}{-\left|y\left(\hat{\beta}_{1}, \hat{\beta}_{2}+w\right)\right| \pm\left|y\left(\hat{\beta}_{1}, \hat{\beta}_{2}\right)\right|} w .
\end{aligned}
$$




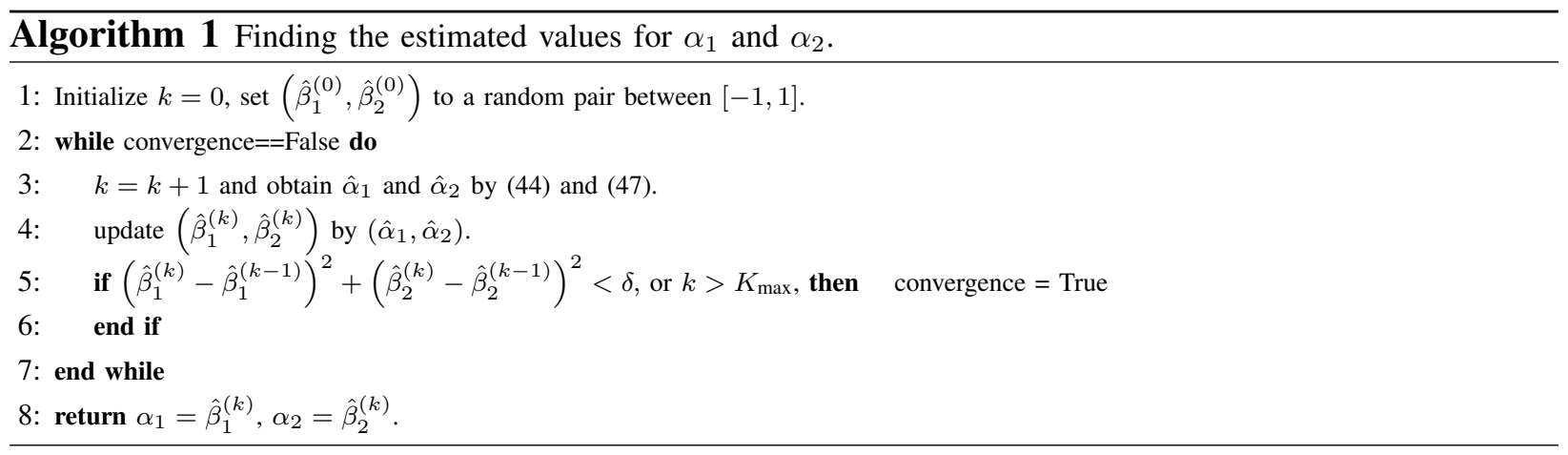

Then, using (38) and (39), the correct solution for $\alpha_{2}$ can be obtained as

$$
\alpha_{2} \approx\left\{\frac{\alpha_{2}^{(i)}+\alpha_{2}^{(j)}}{2}\left|\min _{i, j}\right| \alpha_{2}^{(i)}-\alpha_{2}^{(j)} \mid ; i \in\{1,2\}, j \in\{3,4\}\right\} .
$$

Remark 1: The random choices for $\hat{\beta}_{1}$ and $\hat{\beta}_{2}$ may leads to the null points of the sinc functions in (31), which occurs at $\hat{\beta}_{1}=\alpha_{1} \pm \frac{q}{K_{x}}$ and $\hat{\beta}_{2}=\alpha_{2} \pm \frac{q}{K_{y}}$, where $q \in \mathbb{N}$. For these unfortunate initial values, the received signals at the LISBT are zero, and hence we cannot estimate $\alpha_{1}$ and $\alpha_{2}$. Since the initial values are chosen randomly, the probability of occurrence at the null points of the sinc functions are close to zero. However, if this happens, we change the initial values from $\hat{\beta}_{1}$ and $\hat{\beta}_{2}$ to $\hat{\beta}_{1}+\frac{1}{2 K_{x}}$ and $\hat{\beta}_{2}+\frac{1}{2 K_{y}}$, respectively, to move from the nulls to their closest peaks.

\section{B. Channel Estimation in the Presence of Noise}

The proposed scheme in the previous subsection perfectly estimates the channel parameters for the ideal case when there is no noise at the receiver. In the presence of noise at the receiver, the estimated values in (37) and (40) have errors. In this section, we utilize the estimation scheme introduced in Sec. V-A to propose an iterative algorithm to decrease the channel estimation error due to the noise.

In the presence of noise, if the user sends the pilot $x_{p}=\sqrt{P_{p}}$ to the LISBT, the received signal at the LISBT, denoted by $\hat{y}$, is given by

$$
\hat{y}\left(\beta_{1}, \beta_{2}\right)=\sqrt{P_{p}} \times H^{(f f)}\left(\beta_{1}, \beta_{2}\right)+n,
$$

where $n$ denotes the additive white Gaussian noise (AWGN) at the LISBT.

The proposed iterative algorithm for noisy channel estimation is presented in Algorithm 1, in which index $k$ is used to denote the $k$-th iteration. This algorithm works as follows. As the 
starting point for the iterative algorithm, we choose $\left(\hat{\beta}_{1}^{(0)}, \hat{\beta}_{2}{ }^{(0)}\right)$ as a random pair between $[-1,1]$. In each iteration, the user sends five pilots to the LISBT. Then, the LISBT estimates $\alpha_{1}$ and $\alpha_{2}$ based on the received pilots, as follows. In the $k$-th iteration, similar to (35) and (36), four solutions for $\alpha_{1}$ can be obtained as

$$
\begin{aligned}
& \alpha_{1}^{(1) /(2)}=\hat{\beta}_{1}{ }^{(k-1)}+\frac{\left|\hat{y}\left(\hat{\beta}_{1}{ }^{(k-1)}+v, \hat{\beta}_{2}{ }^{(k-1)}\right)\right|}{\left|\hat{y}\left(\hat{\beta}_{1}^{(k-1)}+v, \hat{\beta}_{2}^{(k-1)}\right)\right| \pm\left|\hat{y}\left(\hat{\beta}^{(k-1)}, \hat{\beta}^{(k-1)}\right)\right|} v, \\
& \alpha_{1}^{(3) /(4)}=\hat{\beta}_{1}{ }^{(k-1)}+\frac{\left|\hat{y}\left(\hat{\beta}_{1}{ }^{(k-1)}-v, \hat{\beta}_{2}{ }^{(k-1)}\right)\right|}{-\left|\hat{y}\left(\hat{\beta}_{1}{ }^{(k-1)}-v, \hat{\beta}_{2}{ }^{(k-1)}\right)\right| \pm\left|\hat{y}\left(\hat{\beta}_{1}{ }^{(k-1)}, \hat{\beta}_{2}{ }^{(k-1)}\right)\right|} v .
\end{aligned}
$$

Similar to (37), we obtain $\alpha_{1}$ as the average of two answers with the minimum difference.

$$
\hat{\alpha}_{1}=\left\{\frac{\alpha_{1}^{(i)}+\alpha_{1}^{(j)}}{2}\left|\min _{i, j}\right| \alpha_{1}^{(i)}-\alpha_{1}^{(j)} \mid ; i \in\{1,2\}, j \in\{3,4\}\right\} .
$$

Similarly, four answers for $\alpha_{2}$ can be obtained as

$$
\begin{aligned}
& \alpha_{2}^{(1) /(2)}=\hat{\beta}_{2}^{(k-1)}+\frac{\left|\hat{y}\left(\hat{\beta}_{1}^{(k-1)}, \hat{\beta}_{2}^{(k-1)}+w\right)\right|}{\left|\hat{y}\left(\hat{\beta}_{1}^{(k-1)}, \hat{\beta}_{2}^{(k-1)}+w\right)\right| \pm\left|\hat{y}\left(\hat{\beta}_{1}{ }^{(k-1)}, \hat{\beta}_{2}{ }^{(k-1)}\right)\right|} w, \\
& \alpha_{2}^{(3) /(4)}=\hat{\beta}_{2}^{(k-1)}+\frac{\left|\hat{y}\left(\hat{\beta}^{(k-1)}, \hat{\beta}_{2}^{(k-1)}-w\right)\right|}{-\left|\hat{y}\left(\hat{\beta}_{1}^{(k-1)}, \hat{\beta}_{2}^{(k-1)}-w\right)\right| \pm\left|\hat{y}\left(\hat{\beta}^{(k-1)}, \hat{\beta}_{2}^{(k-1)}\right)\right|} w .
\end{aligned}
$$

Then, $\hat{\alpha}_{2}$ can be obtained as

$$
\hat{\alpha}_{2}=\left\{\frac{\alpha_{2}^{(i)}+\alpha_{2}^{(j)}}{2}\left|\min _{i, j}\right| \alpha_{2}^{(i)}-\alpha_{2}^{(j)} \mid ; i \in\{1,2\}, j \in\{3,4\}\right\} .
$$

Now, the process of updating $\left(\hat{\beta}_{1}{ }^{(k)}, \hat{\beta}_{2}{ }^{(k)}\right)$ by $\left(\hat{\alpha}_{1}, \hat{\alpha}_{2}\right)$ continues until convergence occurs. The convergence criterion is checked at the end of each iteration by observing whether the following inequality holds

$$
\left(\hat{\beta}_{1}{ }^{(k)}-\hat{\beta}_{1}^{(k-1)}\right)^{2}+\left(\hat{\beta}_{2}^{(k)}-\hat{\beta}_{2}^{(k-1)}\right)^{2}<\delta
$$

where $\delta$ is a small threshold. If (48) holds, the iteration stops and $\left(\hat{\beta}_{1}{ }^{(k)}, \hat{\beta}_{2}{ }^{(k)}\right)$ are adopted as the correct estimations. 


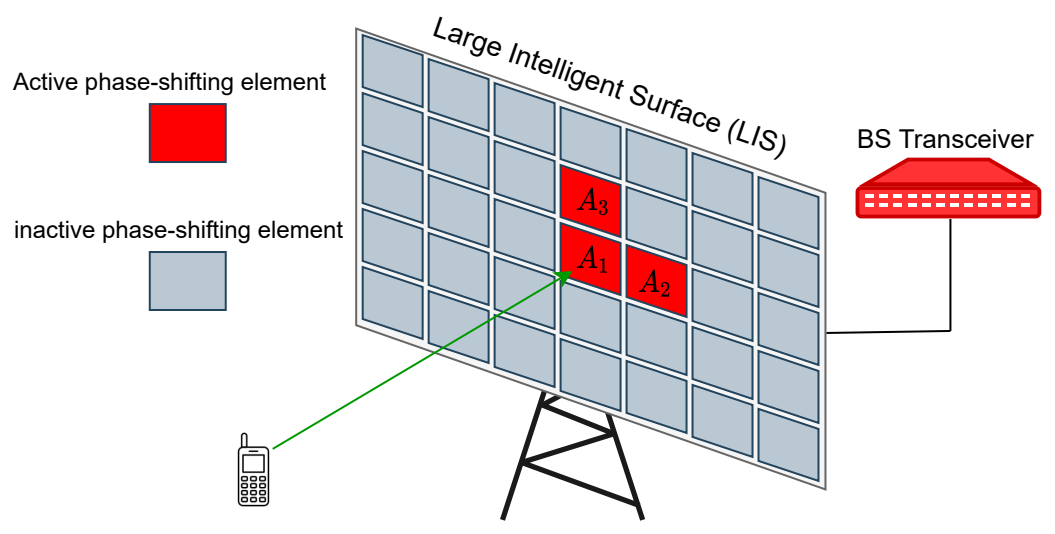

Figure 5. Three radiating elements placed at $(0,0),\left(d_{r}, 0\right)$, and $\left(0, d_{r}\right)$ are activated in three different time slot to provide initial values for the iterative algorithm.

\section{Initial values for the Iterative Algorithm}

The performance of the proposed iterative algorithm depends on the initial values of $\hat{\beta}_{1}^{(0)}, \hat{\beta}_{2}^{(0)}$. When the initial values of the iterative algorithm are random, it means that the LISBT focuses the reception of the pilot signals from random directions. As a result, the received signal at the LISBT may be comparable to the noise, which degrades the performance of the iterative algorithm. On the other hand, when the initial values are close to $\alpha_{1}$ and $\alpha_{2}$, the iterative algorithm can accurately estimate $\alpha_{1}$ and $\alpha_{2}$. In the following, we propose a simple method to provide the initial values of $\hat{\beta}_{1}^{(0)}, \hat{\beta}_{2}^{(0)}$ close to $\alpha_{1}$ and $\alpha_{2}$.

In order to provide initial values, the user sends three pilot signals in three different time slots. In each time slot, the LISBT activates only one phase-shifting element. In the $i$-th time slot, the LISBT activates the $A_{i}$-th element, where $A_{1}, A_{2}$, and $A_{3}$ are placed at $(0,0),\left(d_{r}, 0\right)$, and $\left(0, d_{r}\right)$, respectively, see Fig. 5 . Let $d_{i}, i \in\{1,2,3\}$, denote the distance between the user and the $A_{i}$-th activated element. The user sends the pilot $x_{p}=\sqrt{P_{p}}$ to the LISBT, where $P_{p}$ is the pilot transmit power. According to (1), the received signal at the LISBT in the $i$-th time slot, denoted by $y_{i}$, is given by

$$
y_{i}=\sqrt{P_{p}} \frac{\sqrt{F_{i}} \lambda}{4 \pi d_{i}} e^{-j k_{0} d_{i}}+n_{i}, \quad i \in\{1,2,3\},
$$

where $F_{i}$ accounts for the power radiation pattern of the $A_{i}$-th activated element and the user's antenna and $n_{i}$ denotes the AWGN at the $i$-th time slot. Since three active elements are close to each other, $F_{i}$ is the same for these elements, i.e., $F_{i}=F$. 
From (12), the distance between three active elements and the user can be written as

$$
\begin{aligned}
& d_{1}=d_{0}, \\
& d_{2} \approx d_{0}-d_{r} \alpha_{1}, \\
& d_{3} \approx d_{0}-d_{r} \alpha_{2} .
\end{aligned}
$$

Substituting (50)-(52) into (49) and using the phase of the received signal at the active elements, we can estimate $\alpha_{1}$ and $\alpha_{2}$ as follows

$$
\begin{aligned}
& \hat{\alpha}_{1}=\frac{\measuredangle y_{2}-\measuredangle y_{1}}{k_{0} d_{r}}, \\
& \hat{\alpha}_{2}=\frac{\measuredangle y_{3}-\measuredangle y_{1}}{k_{0} d_{r}},
\end{aligned}
$$

where $\measuredangle y_{i}$ denotes the phase of $y_{i}$. It is worth noting that due to the noise, $\hat{\alpha}_{1}$ and $\hat{\alpha}_{2}$ are not accurate enough. Since the LISBT with infinitely many antennas provides super-resolution beamforming, a small error in the estimation values can degrade the performance of the system during data transmission. Therefore, these estimated values can only provide good initial values for the iterative algorithm.

Remark 2: For the near-field region, we use (53) and (54) to provide initial values for the iterative algorithm. Then, we apply Algorithm 1 to the individual tiles to estimate $\alpha_{1_{n_{x} n_{y}}}$ and $\alpha_{2_{n_{x} n_{y}}}, \forall n_{x}, n_{y}$. Finally, we use (26) to configure the phase-shifting elements of all tiles, in which $\beta_{0_{n_{x} n_{y}}}$ is the phase of the channel between $\left(n_{x}, n_{y}\right)$-th tile and the user.

\section{NUMERICAL RESUltS}

In this section, we provide the numerical results to evaluate the performance of the proposed channel estimation algorithm.

\section{A. Settings of Numerical Experiments}

In this subsection, we explain the parameter setup of the numerical experiments. The simulation results have been averaged over 1000 random channel realization. In each channel realizations, while the distance between the user and the center of the LISBT is fixed, the elevation and azimuth angles of the LoS path follow the uniform distribution, i.e., $\theta \sim U(0, \pi / 2), \phi \sim U(0,2 \pi)$. In addition to the LoS path, we assume that there are 4 NLoS paths due to scatters between the user and the LISBT. The elevation and azimuth angles of each NLoS path from those scatters 
Table I. A list of system parameters for numerical experiments.

\begin{tabular}{c||c||c}
\hline Parameters & Values & Description \\
\hline$f_{c}$ & $30 \mathrm{GHz}$ & Carrier frequency \\
\hline$\lambda$ & $1 \mathrm{~cm}$ & Wavelength \\
\hline$M_{x}$ & 257 & Number of antenna along $x$ axis \\
\hline$M_{y}$ & 257 & Number of antenna along $y$ axis \\
\hline$d_{r}$ & $\lambda / 4$ & Unit element spacing \\
\hline$L_{e}$ & $0.8 d_{r}$ & Width and length of each phase-shifting element \\
\hline$P$ & $30 \mathrm{dBm}$ & Transmission power of the LISBT during data transmission \\
\hline$N_{0}$ & $-115 \mathrm{dBm}$ & Noise power for $200 \mathrm{KHz}$ \\
\hline
\end{tabular}

to the center of LISBT follow the uniform distribution. Moreover, we model the path coefficient of each NLoS path as a complex Gaussian random variable, i.e., $\mathcal{C N}\left(0, \sigma^{2}\right)$, where $\sigma^{2}$ is 20 $\mathrm{dB}$ weaker than the power of the LoS component [50]. Unless otherwise specified, the system parameters for numerical experiments are listed in Table I.

\section{B. Performance Evaluation}

In this section, we present the numerical results for the proposed algorithm and make comparisons with other channel estimation schemes. Three benchmark schemes are considered for comparison, including a hierarchical search scheme, a CS-based channel estimation scheme, and localization-based channel estimation.

Benchmark Scheme 1: In the hierarchical search scheme, proposed in [19], closed-form expressions are provided to generate a codebook consisting of codewords with different beam widths. In this scheme, joint sub-array and deactivation approach is exploited to design a binary-tree codebook. The pilot overhead of this hierarchical scheme is given by $2 \log _{2}(M)$, where $M$ is the number of phase-shifting elements at the LISBT.

Benchmark Scheme 2: In the CS-based channel estimation scheme, proposed in [25], the problem is formulated as a sparse signal recovery problem. Then, the problem is solved by the orthogonal matching pursuit algorithm employing non-uniformly quantized angle grids. The pilot overhead of this scheme is given by $\mathcal{O}(L \ln (M))$, where $L$ is the number of non-zero spatial channel paths. 


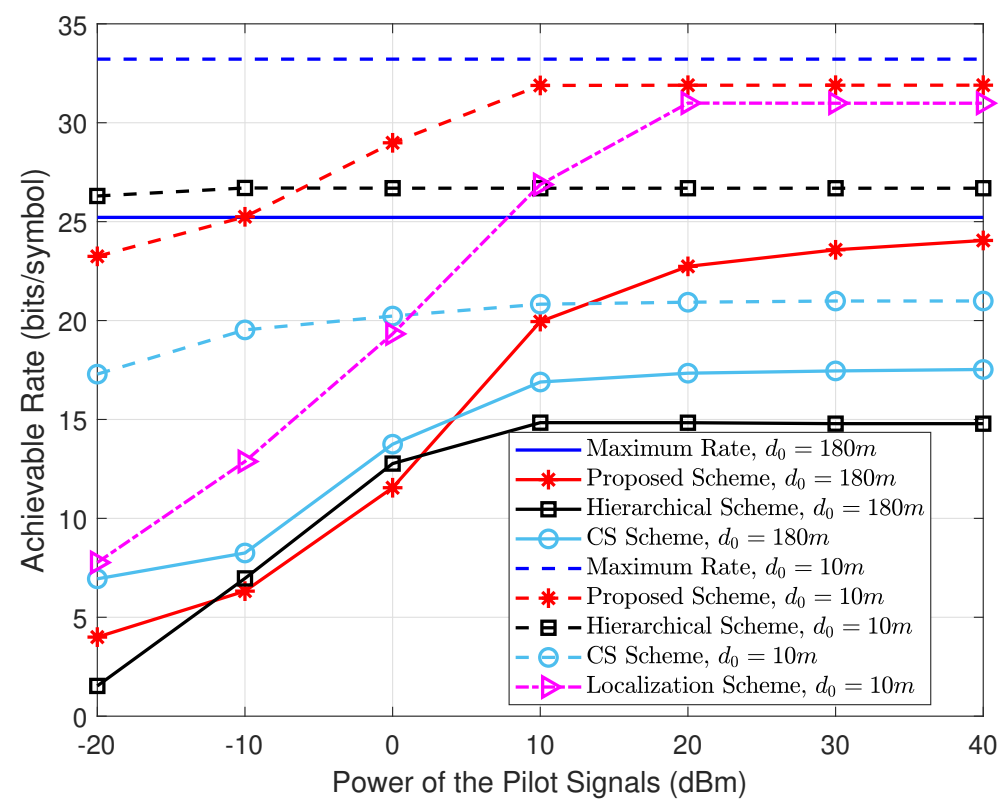

Figure 6. Achievable rate vs. the transmit power of the pilot signals of the proposed scheme and benchmark schemes for both far-field and near-field regions of the LISBT when the number of the pilot signals is fixed to 23 .

Benchmark Scheme 3: In the near-field of the LISBT, the channel is characterized by the location of the user. Therefore, we consider a localization scheme to estimate the channel by the location parameters. In [41], the location of a single antenna transmitter in the near-field is retrieved from the incident spherical wavefront.

The LISBT uses the acquired CSI during the channel estimation period to maximize the received data rate by the user. Therefore, we consider the achieved data rate by the user using the acquired CSI as a performance metric. The achieved data rate is calculated by

$$
R=\log _{2}\left(1+\frac{P_{r}}{N_{0}}\right),
$$

where $N_{0}$ is the AWGN power and $P_{r}$ is the received power at the user in (3) which depends on the phase configuration at the LIS. It is worthwhile to note that the imperfect CSI is used for the configuration of the elements of the LISBT, whereas a high quality CSI of the scalar end-to-end channel (including beamforming at the transmitter) will be acquired at the user with almost perfect phase estimation to enable coherent communication.

Fig. 6 illustrates the achieved data rate of the proposed scheme and the benchmark schemes as a function of the transmission power of the pilot signals. In addition, in this figure, we compare the performance of the proposed scheme with the benchmark schemes in both the far-field and 


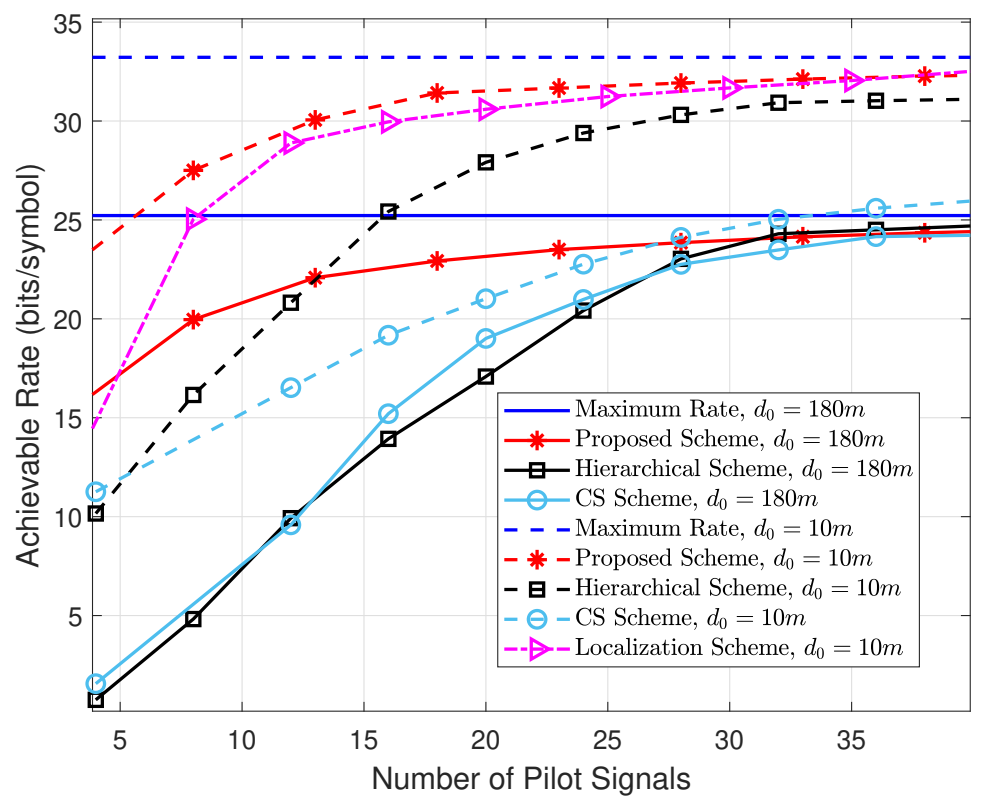

Figure 7. Achievable rate vs. the number of pilot signals of the proposed scheme and benchmark schemes for both far-filed and near-field regions of the LISBT when the transmit power of the pilot signals is fixed to $20 \mathrm{dBm}$.

near-field regions of the LISBT. In the far-field region of the LISBT, we set the maximum number of iteration of the proposed scheme to 4 . For the near-field, we partitioned the LIS into 4 tiles. For each tile, we set the number of iteration to one. Since each iteration requires 5 pilot signals and three pilot signals are required to provide the initial values, the maximum total number of pilot signals is 23. It can be observed from Fig. 6 that when the power of the pilot signals is low, the noise is comparable to the received pilot signals, and hence the proposed scheme cannot estimate the unknown parameters accurately. However, when the power of the pilot signal increases, the received pilot signals are much stronger than the noise, and hence the estimation error decreases. As illustrated in Fig. 6, the proposed scheme, in general, achieves a significant gain over the other benchmark schemes since it exploits the specific structure of the radiated beam (the sinc function).

In Fig. 7, we show the achieved data rate of the proposed scheme and the benchmark schemes as a function of the number of pilot signals. In this figure, we fix the transmit power of the pilot signals to $20 \mathrm{dBm}$. We observe from Fig. 7 that the achieved data rate of the proposed and benchmark schemes increases with the number of pilot signals. In the far-field region of the LISBT, the proposed, hierarchical, and CS schemes can approximately achieve the maximum 


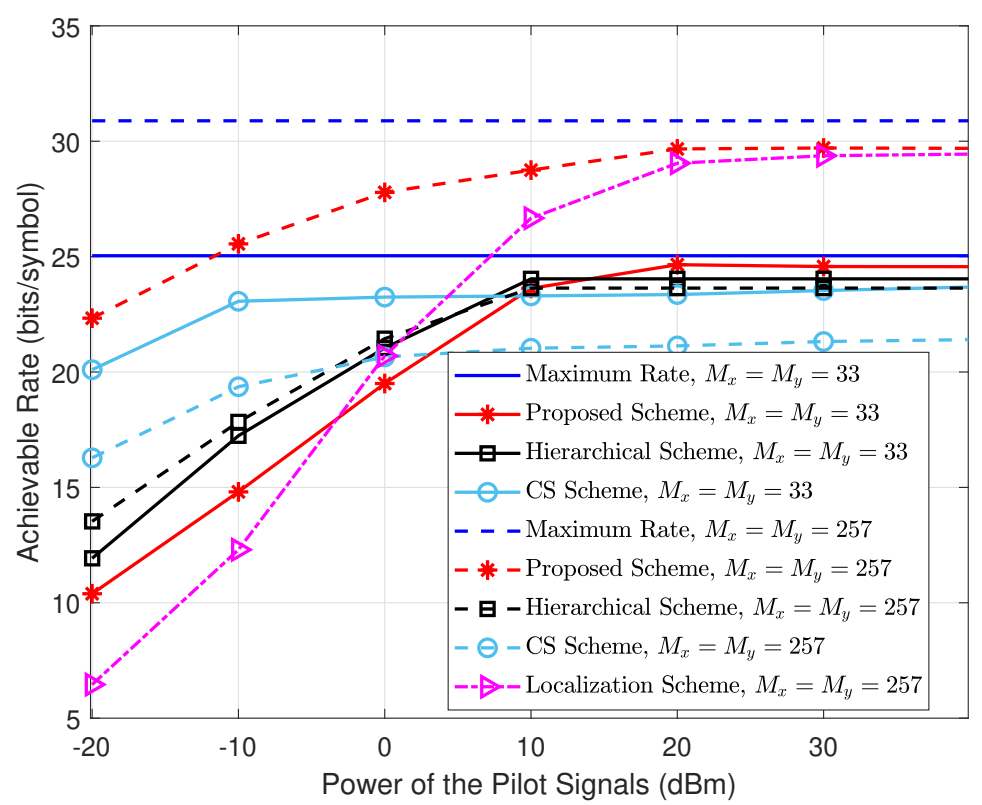

Figure 8. Achievable rate vs. the transmit power of the pilot signals of the proposed scheme and the benchmark schemes for different number of phase-shifting elements when the number of the pilot signals is fixed to 23 .

rate when the number of pilot signals is more than 30. In the near-field region of the LISBT, the achieved data rate of the CS scheme cannot increase more than a certain value due to the assumption of quantized values for $\alpha_{1}$ and $\alpha_{2}$. As illustrated in Fig. 7, the proposed scheme outperforms all benchmark schemes for the different number of pilot signals.

Fig. 8 compares the achieved data rate by the proposed scheme with the benchmark schemes for two different numbers of phase-shifting elements at the LISBT. Similar to Fig. 6, the number of pilot signals is fixed to 23. Assuming perfect CSI, the achieved data rate has to increase with $M$. Nevertheless, the performance of the hierarchical scheme does not change with $M$. This is due to the fact that when $M$ increases, the signal beam width is narrower, and hence, more accurate estimations for $\alpha_{1}$ and $\alpha_{2}$ are required, which is not feasible with a low number of pilot signals. In addition, the performance of the CS scheme decreases with $M$ since the pilot overhead of the $\mathrm{CS}$ scheme is $\mathcal{O}(L \ln (M))$. On the other hand, the achieved data rate of the proposed scheme increases with $M$, since accurate estimation for $\alpha_{1}$ and $\alpha_{2}$ can be obtained by the proposed scheme.

Fig. 9 illustrates the achieved data rate of the proposed scheme and the benchmark schemes as a function of the power difference of LoS and NLoS paths. The horizontal axis of this figure 


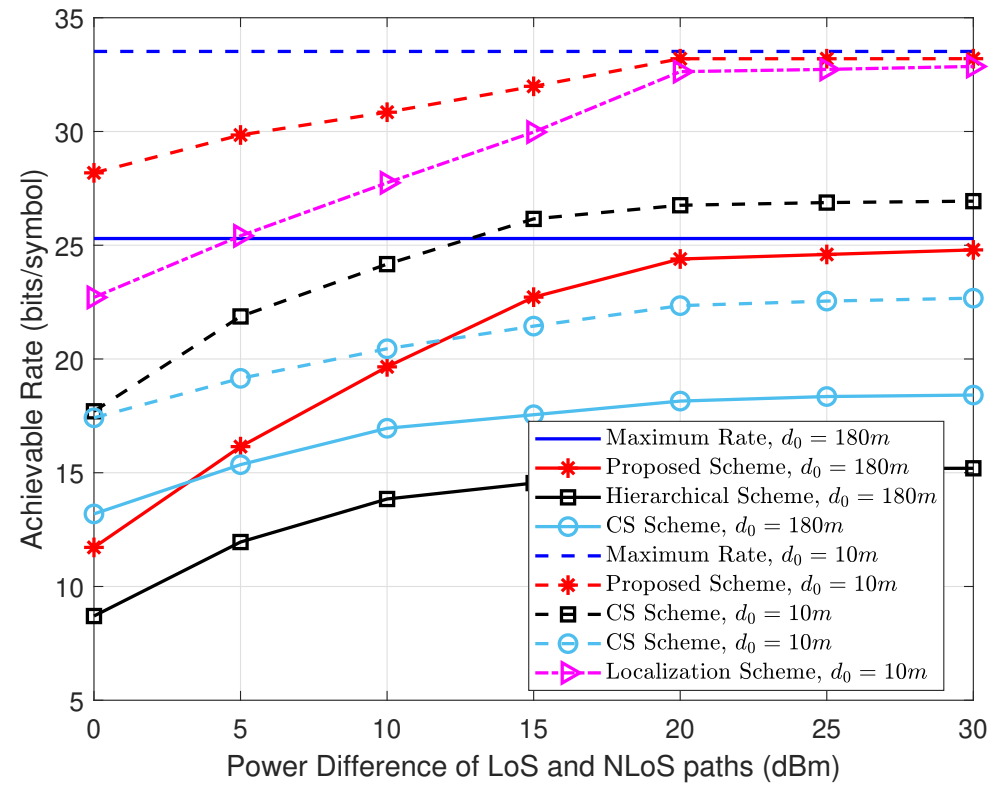

Figure 9. Achievable rate vs. power difference of LoS and NLoS paths of the proposed scheme and benchmark schemes for both far-field and near-field of the LISBT when the transmit power and the number of the pilot signals are fixed to $20 \mathrm{dBm}$ and 23 , respectively.

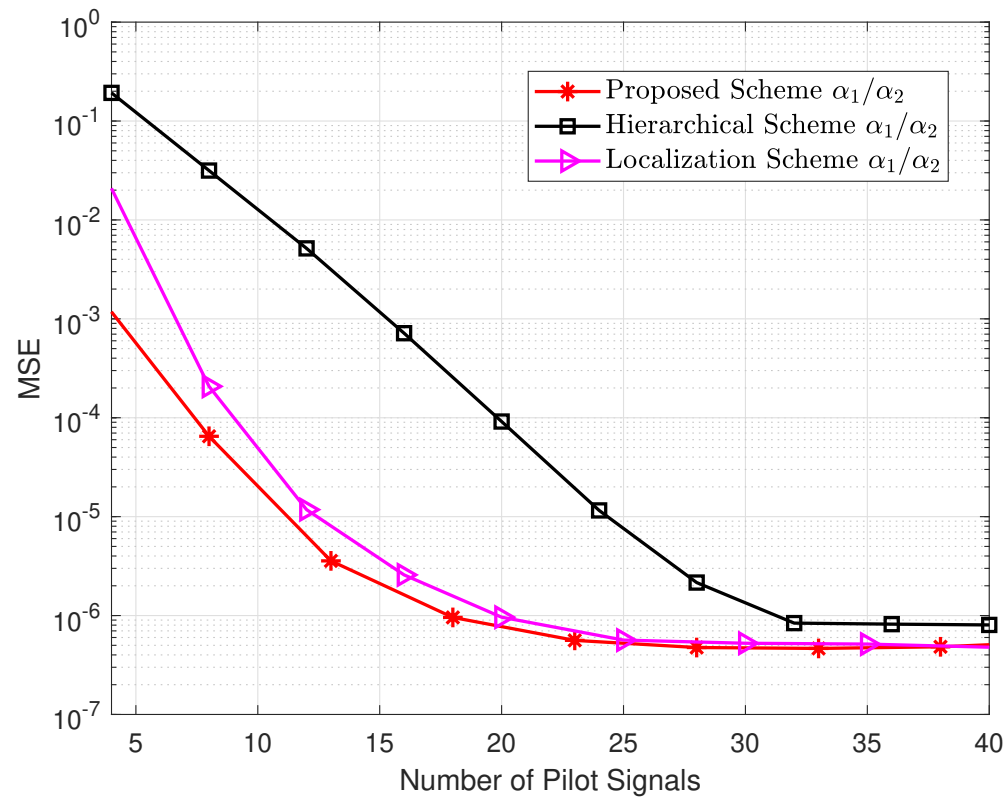

Figure 10. MSE vs. the number of pilot signals of the proposed scheme and the hierarchical scheme when the transmit power and the distance between the LISBT and the user are fixed to $20 \mathrm{dBm}$ and $25 \mathrm{~m}$, respectively. 
indicates that how much the power of NLoS path components is weaker than the power of the LoS component. In this figure, the transmit power and the number of the pilot signals are fixed to $20 \mathrm{dBm}$ and 23 , respectively. In the proposed scheme, we consider the effect of NLoS path components as noise. As a result, when the power of NLoS path components decreases, the proposed scheme can estimate $\alpha_{1}$ and $\alpha_{2}$ more accurately. As shown in Fig. 9, the proposed scheme outperforms all benchmark schemes even when the power of NLoS path components is comparable to the power of LoS path component.

In Fig. 10, we compare the convergence behavior of the proposed scheme with the hierarchical and localization schemes. We consider the mean square error (MSE) to study the convergence behaviour, which is defined by $\operatorname{MSE}_{i}=\mathbb{E}\left\{\left|\alpha_{i}-\hat{\alpha}_{i}\right|^{2}\right\}, \forall i \in\{1,2\}$. In this figure, we fix the transmit power and the distance between the LISBT and the user to $20 \mathrm{dBm}$ and $25 \mathrm{~m}$, respectively. Since the CS scheme estimates the whole channel, not the AoA/AoD, we compare the proposed scheme only with the hierarchical and localization schemes. We observe from Fig. 10 that when the number of pilot signals increases, the MSE of the proposed scheme and the benchmark schemes decreases. In the hierarchical scheme, when the number of pilot signals increases, narrower beam widths are used to estimate $\alpha_{1}$ and $\alpha_{2}$ more accurately. However, since $M_{x}=M_{y}=257$, after $\left\lfloor\log _{2} 257\right\rfloor=8$ iteration, the MSE of the hierarchical scheme does not decrease. Fig. 10 suggests that the proposed scheme and localization scheme can accurately estimate $\alpha_{1}$ and $\alpha_{2}$ when the number of pilot signals is more than 23 (4 iterations in Algorithm 1).

\section{CONCLUSION}

In this paper, we proposed a channel estimation scheme for the LISBT-assisted wireless communication system. In the far-field region of the LISBT, we modeled the channel based on the path parameters of the system. It is shown that only two path parameters are required to be estimated to obtain the optimal phase shifts for all phase-shifting elements of the LISBT. For the near-field, we first partitioned the IRS into tiles. Then, the channel was modeled as the superposition of the channels through the individual tiles. Moreover, only two parameters are required to be estimated for each tile. We showed that the proposed scheme requires only five pilots to perfectly estimate the unknown parameters in the absence of noise. In the presence of the noise, an iterative algorithm was presented to estimate the unknown path parameters. The 
simulation results verified that the proposed scheme achieves significant performance gains over existing channel estimation schemes.

\section{APPENDIX}

\section{A. Proof of Lemma 1}

We start the proof by substituting the linear phase shift in (20) into (18). We have

$$
\begin{aligned}
H^{(f f)}\left(\beta_{1}, \beta_{2}\right) & \approx\left(\frac{\sqrt{F} \lambda e^{-j k_{0} d_{0}}}{4 \pi d_{0}}\right) \sum_{m_{x}=-\frac{M_{x}-1}{2}}^{\frac{M_{x}-1}{2}} \sum_{m_{y}=-\frac{M_{y}-1}{2}}^{\frac{M_{y}-1}{2}} e^{j k_{0} d_{r}\left(m_{x}\left(\alpha_{1}-\beta_{1}\right)+m_{y}\left(\alpha_{2}-\beta_{2}\right)\right)} \\
& \approx\left(\frac{\sqrt{F} \lambda e^{-j k_{0} d_{0}}}{4 \pi d_{0}}\right) \sum_{m_{x}=-\frac{M_{x}-1}{2}}^{\frac{M_{x}-1}{2}} e^{j k_{0} d_{r}\left(m_{x}\left(\alpha_{1}-\beta_{1}\right)\right)} \sum_{m_{y}=-\frac{M_{y}-1}{2}}^{\frac{M_{y}-1}{2}} e^{j k_{0} d_{r}\left(m_{y}\left(\alpha_{2}-\beta_{2}\right)\right)} .
\end{aligned}
$$

Using the sum of terms in a geometric progression, we can write

$$
\sum_{m=-\frac{M-1}{2}}^{\frac{M-1}{2}} e^{j m a}=\frac{e^{-j\left(\frac{M-1}{2}\right) a}\left(1-e^{j M a}\right)}{1-e^{j a}}=\frac{\sin (M a / 2)}{\sin (a / 2)} .
$$

Now, using (58) in (57), we have

$$
H^{(f f)}\left(\beta_{1}, \beta_{2}\right) \approx\left(\frac{\sqrt{F} \lambda e^{-j k_{0} d_{0}}}{4 \pi d_{0}}\right) \frac{\sin \left(M_{x} \frac{k_{0} d_{r}}{2}\left(\alpha_{1}-\beta_{1}\right)\right)}{\sin \left(\frac{k_{0} d_{r}}{2}\left(\alpha_{1}-\beta_{1}\right)\right)} \times \frac{\sin \left(M_{y} \frac{k_{0} d_{r}}{2}\left(\alpha_{2}-\beta_{2}\right)\right)}{\sin \left(\frac{k_{0} d_{r}}{2}\left(\alpha_{2}-\beta_{2}\right)\right)} .
$$

Substituting $M_{x}=L_{x} / d_{r}$ and $M_{y}=L_{y} / d_{r}$ into (21) completes the proof.

\section{REFERENCES}

[1] C. Chaccour, M. N. Soorki, W. Saad, M. Bennis, P. Popovski, and M. Debbah, "Seven defining features of terahertz (THz) wireless systems: A fellowship of communication and sensing," arXiv preprint arXiv:2102.07668, 2021.

[2] W. Tang, X. Chen, M. Z. Chen, J. Y. Dai, Y. Han, M. Di Renzo, S. Jin, Q. Cheng, and T. J. Cui, "Path loss modeling and measurements for reconfigurable intelligent surfaces in the millimeter-wave frequency band," arXiv preprint arXiv:2101.08607, 2021.

[3] E. Basar, M. Di Renzo, J. De Rosny, M. Debbah, M. S. Alouini, and R. Zhang, "Terahertz massive MIMO with holographic reconfigurable intelligent surfaces," IEEE Trans. Wireless Commun., vol. , no. , pp. 4732-4750, Mar. 2021.

[4] V. Jamali, A. M. Tulino, G. Fischer, R. Müller, and R. Schober, "Intelligent Surface-Aided Transmitter Architectures for Millimeter-Wave Ultra Massive MIMO Systems,” IEEE Open J. Commun. Soc., vol. 2, pp. 144-167, Dec. 2020.

[5] L.Wei, R. Q. Hu, Y. Qian, and G.Wu, "Key elements to enable millimeter wave communications for 5G wireless systems," IEEE Trans. Wireless Commun., vol. 21, no. 6, pp. 136-143, Dec. 2014.

[6] E. Larsson, O. Edfors, F. Tufvesson, and T. Marzetta, "Massive MIMO for next generation wireless systems," IEEE Commun. Mag., vol. 52, no. 2, pp. 186-195, Feb. 2014. 
[7] Y. Zhu, G. Zheng and K. -K. Wong, "Stochastic Geometry Analysis of Large Intelligent Surface-Assisted Millimeter Wave Networks," IEEE J. Sel. Areas Commun., vol. 38, no. 8, pp. 1749-1762, Aug. 2020.

[8] E. Björnson, L. Sanguinetti, H. Wymeersch, J. Hoydis, and T. L. Marzetta, "Massive MIMO is a reality—What is next?: Five promising research directions for antenna arrays,” Digit. Signal Process., vol. 94, PP. 3-20, Nov. 2019.

[9] D. Dardari, "Communicating With Large Intelligent Surfaces: Fundamental Limits and Models," IEEE J. Sel. Areas Commun., vol. 38, no. 11 , pp. 2526-2537, Nov. 2020.

[10] C. Huang et al., "Holographic MIMO surfaces for 6G wireless networks: Opportunities, challenges, and trends," IEEE Wireless Commun., vol. 27, no. 5, pp. 118-125, Oct. 2020.

[11] N. Shlezinger, G. C. Alexandropoulos, M. F. Imani, Y. C. Eldar, and D. R. Smith, "Dynamic metasurface antennas for 6G extreme massive MIMO communications,” IEEE Wireless Commun., vol. 28 , no. , PP. 106-113, Jan. 2021.

[12] M. D. Renzo et al, "Smart radio environments empowered by reconfigurable intelligent surfaces: How it works, state of research, and the road ahead," IEEE J. Sel. Areas Commun., vol. 38, no. 11, pp. 2450-2525, Nov. 2020.

[13] Q. Wu, S. Zhang, B. Zheng, C. You, and R. Zhang, "Intelligent reflecting surface aided wireless communications: A tutorial," IEEE Trans. Wireless Commun., vol. 69 , no. 5, PP. 3313-3351, May. 2021.

[14] Q. Wu and R. Zhang, "Intelligent reflecting surface enhanced wireless network via joint active and passive beamforming," IEEE Trans. Wireless Commun., vol. 18, no. 10, pp. 5394-5409, Nov. 2019.

[15] M. Jung, W. Saad, G. Kong, "Performance Analysis of Active Large Intelligent Surfaces (LISs): Uplink Spectral Efficiency and Pilot Training," IEEE Trans. Wireless Commun., vol. 69 , no. 5, PP. 3379-3394, May 2021.

[16] S. Hu, F. Rusek, and O. Edfors, "Beyond massive MIMO: The potential of data transmission with large intelligent surfaces," IEEE Trans. Signal Process., vol. 66, no. 10, pp. 2746-2758, May 2018.

[17] J. Yuan, H. Q. Ngo, and M. Matthaiou, "Towards large intelligent surface (LIS)-based communications," IEEE Trans. Commun., vol. 68, no. 10, pp. 6568-6582, Oct. 2020.

[18] S. Hu, F. Rusek, and O. Edfors, "Beyond massive MIMO: The potential of positioning with large intelligent surfaces," IEEE Trans. Signal Process., vol. 66, no. 7, Apr. 2018, pp. 1761-1774.

[19] Z. Xiao, T. He, P. Xia, and X.-G. Xia, "Hierarchical codebook design for beamforming training in millimeter-wave communication,” IEEE Trans. Wireless Commun., vol. 15, no. 5, pp. 3380-3392, May 2016.

[20] F. Dai and J. Wu, "Efficient Broadcasting in Ad Hoc Wireless Networks Using Directional Antennas," IEEE Trans. Parallel Distrib. Syst., vol. 17, no. 4, pp. 335-347, Aug. 2006.

[21] J. Zhang, Y. Huang, Q. Shi, J. Wang, and L. Yang, "Codebook design for beam alignment in millimeter wave communication systems," IEEE Trans. Commun., vol. 65, no. 11, pp. 4980-4995, Nov. 2017.

[22] S. Noh, M. D. Zoltowski, and D. J. Love, "Multi-resolution codebook and adaptive beamforming sequence design for millimeter wave beam alignment," IEEE Trans. Wireless Commun., vol. 16, no. 9, pp. 5689-5701, Sep. 2017.

[23] K. Chen and C. Qi, "Beam training based on dynamic hierarchical codebook for millimeter wave massive MIMO," IEEE Commun. Lett, vol. 23, no. 1, pp. 132-135, Jan. 2019.

[24] J. Lee, G. T. Gil, and Y. H. Lee, "Channel estimation via orthogonal matching pursuit for hybrid MIMO systems in millimeter wave communications," IEEE Trans. Commun., vol. 64, no. 6, pp. 2370-2386, Jun. 2016.

[25] C. R. Tsai, Y. H. Liu, and A. Y. Wu, "Efficient Compressive Channel Estimation for Millimeter-Wave Large-Scale Antenna Systems," IEEE Trans. Signal Process., vol. 66, no. 9, pp. 2414-2428, May 2018.

[26] X. Ma, F. Yang, S. Liu, J. Song, and Z. Han, "Design and optimization on training sequence for mmWave communications: A new approach for sparse channel estimation in massive MIMO,” IEEE J. Sel. Areas Commun., vol. 35, no. 7, pp. 1486-1497, Jul. 2017. 
[27] Y. Peng, Y. Li, and P.Wang, "An enhanced channel estimation method for millimeter wave systems with massive antenna arrays," IEEE Commun. Lett., vol. 19, no. 9, pp. 1592-1595, Sep. 2015.

[28] S. H. Lim, S. Kim, B. Shim, and J.W. Choi, "Efficient Beam Training and Sparse Channel Estimation for Millimeter Wave Communications Under Mobility," IEEE Trans. Commun., vol. 68, no. 10, pp. 6583-6596, Oct. 2020.

[29] S. Pejoski and V. Kafedziski, "Estimation of sparse time dispersive channels in pilot aided OFDM using atomic norm," IEEE Commun. Lett., vol. 4, no. 4, pp. 397-400, Aug. 2015.

[30] H. Chu, L. Zheng, and X. Wang, "Super-resolution mmwave channel estimation for generalized spatial modulation systems," IEEE J. Sel. Topics. Signal Process., vol. 13, no. 6, pp. 1336-1347, Oct. 2019.

[31] M. S'anchez-Fern 'andez, V. Jamali, J. Llorca, and A. Tulino, "Gridless Multidimensional Angle-of-Arrival Estimation for Arbitrary 3D Antenna Arrays," IEEE Trans. Wireless Commun., vol. 20, no. 7, pp. 4748-4764, Jul. 2021.

[32] R. Shafin, L. Liu, Y. Li, A. Wang, and J. Zhang, "Angle and delay estimation for 3-D massive MIMO/FD-MIMO systems based on parametric channel modeling," in IEEE Trans. Wireless Commun., vol. 16, no. 8, pp. 5370-5383, Aug. 2017.

[33] J. He, H. Wymeersch, and M. Juntti, "Channel estimation for RIS-aided mmWave MIMO systems via atomic norm minimization,” in IEEE Trans. Wireless Commun., vol. 20, no. 9, pp. 5786-5797, Sep. 2021.

[34] Q. Nadeem, H. Alwazani, A. Kammoun, A. Chaaban, M. Debbah, and M. S. Alouini, "Millimeter-Wave Based Localization Using a Two-Stage Channel Estimation Relying on Few-Bit ADCs," IEEE Open J. Commun. Soc., vol. 2, pp. 1736-1752, Jul. 2021.

[35] Y. Wang and K. C. Ho, "Unified near-field and far-field localization for AOA and hybrid AOA-TDOA positionings," IEEE Trans. Wireless Commun., vol. 17, no. 2, pp. 1242-1254, Feb. 2018.

[36] H. Wymeersch, J. He, B. Denis, A. Clemente, and M. Juntti, "Radio localization and mapping with reconfigurable intelligent surfaces: Challenges, opportunities, and research directions," IEEE Veh. Technol. Mag., vol. 15, no. 4, pp. 52-61, 2020.

[37] A. Shahmansoori, G. E. Garcia, G. Destino, G. Seco-Granados, and H. Wymeersch, "Position and orientation estimation through millimeter-wave MIMO in 5G systems," IEEE Trans. Wireless Commun., vol. 17, no. 3, pp. 1822-1835, Mar. 2018.

[38] H. Zhang, H. Zhang, B. Di, K. Bian, Z. Han, and L. Song, "Metalocalization: Reconfigurable intelligent surface aided multi-user wireless indoor localization," IEEE Trans. Wireless Commun., early access.

[39] X. Li, E. Leitinger, M. Oskarsson, K. Åström, and F. Tufvesson, "Massive MIMO-based localization and mapping exploiting phase information of multipath components," IEEE Trans. Wireless Commun., vol. 18, no. 9, pp. 4254-4267, Sep. 2019.

[40] R. Mendrzik, H. Wymeersch, G. Bauch, and Z. Abu-Shaban, "Harnessing NLOS components for position and orientation estimation in 5G millimeter wave MIMO," IEEE Trans. Wireless Commun., vol. 18, no. 1, pp. 93-107, Jan. 2018.

[41] F. Guidi and D. Dardari, "Radio positioning with EM processing of the spherical wavefront," IEEE Trans. Wireless Commun., vol. 20, no. 6, pp. 3571-3586, Jun. 2021.

[42] M. Najafi, V. Jamali, R. Schober, and V. H. Poor, "Physics-based modeling and scalable optimization of large intelligent reflecting surfaces (extended version)," pp. 1-38, Apr. 2020, arXiv:2004.12957. [Online]. Available: https://arxiv.org/abs/2004.12957

[43] L. Pan, L. Liang, W. Xu, and X. Dong, "Framework of channel estimation for hybrid analog-and-digital processing enabled massive MIMO communications," IEEE Trans. Commun., vol. 66, no. 9, pp. 3902-3915, Sep. 2018.

[44] M. R. Akdeniz, Y. Liu, M. K. Samimi, S. Sun, S. Rangan, T. S. Rappaport, and E. Erkip, "Millimeter wave channel modeling and cellular capacity evaluation,” IEEE J. Sel. Areas Commun., vol. 32, no. 6, pp. 1164-1179, Jun. 2014.

[45] Z. Muhi-Eldeen, L. Ivrissimtzis, and M. Al-Nuaimi, "Modelling and measurements of millimetre wavelength propagation in urban environments," ET Microw., Antennas Propag.,vol. 4, no. 9, pp. 1300-1309, Sep. 2010.

[46] S. W. Ellingson, "Path loss in reconfigurable intelligent surface-enabled channels," in Proc. IEEE 25th Annu. Int. Symp. Pers., Indoor, Mobile Radio Commun. (PIMRC),, Helsinki, Finland, Sep. 2021. 
[47] C. A. Balanis, Antenna Theory: Analysis and Design. John Wiley \& Sons, 2016.

[48] K. T. Selvan and R. Janaswamy, "Fraunhofer and Fresnel distances: Unified derivation for aperture antennas," IEEE Antennas Propag. Mag., vol. 59, no. 4, pp. 12-15, Aug. 2017.

[49] I. S. Gradshteyn and I. M. Ryzhik, Table of Integrals, Series, and Products., San Diego, CA: Academic, 1994.

[50] W. Wang, and W. Zhang, "Joint Beam Training and Positioning for Intelligent Reflecting Surfaces Assisted Millimeter Wave Communications," IEEE Trans. Wireless Commun., vol. 20, no. 10, pp. 6282-6297, Oct. 2021. 